\title{
Mobility Under the COVID-19 Pandemic: Asymmetric Effects Across Gender and Age
}

\author{
Francesca Caselli ${ }^{1}$ (1) - Francesco Grigoli ${ }^{1}$. Damiano Sandri ${ }^{1,2}$. \\ Antonio Spilimbergo ${ }^{1,2}$
}

Published online: 22 October 2021

(c) International Monetary Fund 2021, corrected publication 2021

\begin{abstract}
Overall mobility declined during the COVID-19 pandemic because of government lockdowns and voluntary social distancing. Yet, aggregate data mask important heterogeneous effects across segments of the population. Using unique mobility indicators based on anonymized and aggregate data provided by Vodafone for Italy, Portugal, and Spain, we find that lockdowns had a larger impact on the mobility of women and younger cohorts. Younger people also experienced a sharper drop in mobility in response to rising COVID-19 infections. Our findings, which are consistent across estimation methods and robust to a variety of tests, warn about a possible widening of gender and inter-generational inequality.
\end{abstract}

JEL Classification $\mathrm{E} 1 \cdot \mathrm{I} 1 \cdot \mathrm{H} 0$

\section{Introduction}

The COVID-19 pandemic dramatically reduced people's mobility. This was due in part to the lockdown measures that governments adopted to reduce personal contacts, including travel restrictions, school, and business closures, and stay-at-home orders. Mobility also declined because people voluntarily reduced social interactions

Francesca Caselli

fcaselli@imf.org

Francesco Grigoli

fgrigoli@imf.org

Damiano Sandri

dsandri@imf.org

Antonio Spilimbergo

aspilimbergo@imf.org

1 IMF, Washington, USA

2 CEPR, London, UK 
out of fear of contracting the virus. The literature has documented these effects using a broad range of aggregate mobility indicators provided by private companies such as Google, Apple, and SafeGraph (Chetty et al. 2020; Goolsbee et al. 2020; Glaeser et al. 2020; Maloney and Taskin 2020).

This paper innovates relatively to the existing studies by showing that mobility patterns differed considerably across gender and age groups. Our analysis is based on novel and confidential mobility indicators provided by Vodafone for Italy, Portugal, and Spain at the provincial level. These data offer the unique advantage of disaggregating mobility information across gender and age groups, making it possible to uncover important heterogeneous reactions to the pandemic and lockdown measures. This paper makes several contributions.

First, the analysis contributes to the growing evidence about the disproportionate impact of the COVID-19 crisis on women. This literature finds that some home production that in normal times can be outsourced had to be performed within the household during the pandemic. And this burden fell disproportionately on women. Hupkau and Petrangolo (2020), for example, find that in UK households women took on a larger share of increased childcare needs, even though fathers became the primary childcare providers in an important share of households. Alon et al. (2020) show that, contrary to past recessions, the current crisis led to a stronger increase in female unemployment in the USA. This is because women are more likely to care for children when schools are closed and because they are employed in sectors more severely hit by the pandemic, such as restaurants and personal care. Survey data also suggest that women face an unequal burden in caring for children when schools are closed and are at a higher risk of facing a reduction in working hours (Adams et al. 2020; Sevilla and Smith 2020). In this paper, we document that the disproportional impact of the crisis on women is also visible in mobility patterns and examine the causes of this differential.

In this regard, this paper also contributes to the literature on the determinants of labor force participation. School closures and other lockdown measures reduce mobility in different ways across gender and thus have a differential effect on labor supply. Previous studies have found that exogenous changes in the length of school schedule impact female labor force participation (Berthelon et al. 2015). We provide high-frequency, complementary evidence.

Second, this paper documents considerable heterogeneity in the impact of lockdowns across age groups. These findings are quite relevant for the ongoing debate on the distribution of costs and benefits across generations (Belot et al. 2020; Glover et al. 2020). While lockdowns protect mainly older people that are more likely to develop serious medical conditions from COVID-19, they impose economic costs especially on working age people that rely on labor income to support consumption.

Third, the heterogeneous impact of lockdowns across age can shed light on the scarring effects from the crisis. For instance, a given reduction in aggregate mobility has different long-term effects if it is concentrated on the young. Oreopoulos et al. (2012) find that individuals entering the labor force during a recession suffer from substantial and persistent loss of earning; they also document that graduates who can switch quickly to better firms suffer less. So a forced reduction in mobility has a particularly adverse and persistent effect if it reduces the mobility of young. 
Recessions (2021) document that workers that face unemployment spells experience earnings losses of around 15 percent once they take a new job. These results on the cyclicality of earnings are broadly consistent with Huckfeldt (2018) and Gertler et al. (2020).

Fourth, heterogeneous effects across gender and age raise important methodological considerations. Lumping all groups together in estimating the effects of containment measures as done in most studies could lead to aggregation bias and mismeasurement problems.

Fifth, in the presence of repeated infection waves in several countries (as we write, many countries are experiencing a strong resurgence) and in the context of an already weak economic activity, authorities need to consider more nuanced containment measures. To design these measures, authorities should consider the heterogeneous effects on different groups. This perspective is particularly important because the health risks posed by COVID-19 are very heterogeneous across age, being much more severe for people aged 65 and above. Therefore, some researches have argued for targeted measures to isolate older people without unduly limiting the mobility and employment opportunities of younger people (Acemoglu et al. 2020; Gollier 2020).

Studying the effect of containment measures on mobility is a difficult task because the adoption of lockdowns is an endogenous policy decision. For example, governments are more likely to impose measures when health risks are more acute. At that time, people tend to also reduce mobility voluntarily because they fear being infected or infecting others. This raises the risk of detecting a spurious correlation between lockdowns and mobility. To address the endogeneity concern, we use a regression discontinuity (RD) design that focuses on high-frequency changes in mobility around specific lockdown measures, thereby reducing the risk that other factors may affect mobility at the same time. In addition, we estimate local projections in which we control for pre-existing mobility trends and for the severity of the country's epidemic. We further strengthen the identification by excluding those provinces that experienced early or particularly severe outbreaks. In this case, the analysis looks at the mobility patterns in provinces less affected by COVID-19 for which the adoption of national lockdowns was largely an exogenous event.

The results from the RD design show that the adoption of stay-at-home orders in Italy, Portugal, and Spain led to a sharper contraction in women's mobility relative to men's. Stay-at-home orders coincided in most provinces with school closures. To isolate the impact of school closures on mobility, we narrow the analysis to provinces in Northern Italy that imposed school closures before stay-at-home orders. We find that school closures led already to a considerable gender differential in mobility, highlighting the uneven role of women in caring for children.

The findings based on local projections corroborate the results of the RD analysis, showing that lockdowns had a disproportionate effect on the mobility of women. The differential impact is statistically significant and quantitatively relevant. Among people aged 24 to 45 , a full lockdown - including all measures used by governments during the pandemic - reduces the number of women leaving home by almost 26 percent, against an impact on men of about 21 percent. 
The local projections also allow to examine the effects of rising COVID-19 cases on mobility for a given level of the stringency of lockdowns. This captures the extent to which people decide to voluntarily limit social interactions when the fear of contracting the virus becomes more acute. Examining this aspect is very important because much of the public debate on the need for lockdowns centers on whether people can autonomously change behavior when infection risks arise. The analysis shows that both men and women significantly reduce mobility when infections increase and they do so with equal intensity.

Regarding the differential effects across age, RD designs show that stay-athome orders disproportionately reduce the mobility of working age people, especially those below 45 years of age. Local projections provide additional evidence that lockdowns have a larger impact on younger cohorts. These findings are concerning because younger workers generally rely on labor income to support consumption, while older people have access to personal savings and possibly retirement income. Furthermore, younger workers often have less stable job contracts that are more likely to be terminated during a crisis. Survey-based evidence confirms that younger people have been more likely to suffer an income loss during the pandemic (Belot et al. 2020; Montenovo et al. 2020). The fact that lockdowns impose a disproportionate economic burden on the young - while protecting mostly the old given the higher health risks - calls for policy intervention to ensure inter-generational fairness (Glover et al. 2020).

Using local projections, we also explore if people of different age respond differently to rising infections. Because COVID-19 poses much greater risks for people aged 65 and above, it could be expected that these people are more likely to isolate themselves when infections rise. On the contrary, the analysis shows that younger people reduce mobility more strongly when infections increase. This is consistent with survey level evidence presented by Bordalo et al. (2020), showing that younger people are more alarmed by the risk of contracting COVID-19.

The interpretation of the results is subject to several caveats. First, our narrative assumes that people that reduce mobility in response to lockdowns or rising COVID-19 infections suffer economic losses even though some of them may also work from home. This is based on a reveal preference argument, whereby if people could work from home with no adverse income consequences, they would do so also before the spread of COVID-19 and the introduction of lockdowns. Yet, we do not have income data to quantify the impact of lower mobility on earnings. Second, it must be recognized that people that stayed at home during the pandemic were also less likely to contract the virus. Therefore, a comprehensive welfare assessment should trade off the income losses due to reduced mobility with the reduction in the infection risks. Third, based on data availability, the analysis focuses on the impact of the first round of lockdowns in the spring of 2020. In future work, it would be helpful to explore if subsequent lockdowns continued to have the same heterogeneous effects documented in our study.

The paper is structured as follows. Section 2 presents background information on the COVID-19 crises in Italy, Portugal, and Spain, and the relative containment measures. Section 3 describes the data provided by Vodafone. Sections 4 
and 5 present the analysis of the mobility patterns by gender and age, respectively. Section 6 examines the robustness of the results. Section 7 concludes.

\section{The COVID-19 Crises in Italy, Portugal, and Spain}

In this section, we describe the evolution of the COVID-19 crises in Italy, Portugal, and Spain and the lockdown measures adopted at the local and national level.

\subsection{Italy}

Italy was among the first countries to be hit by COVID-19 after China. On January 31, the Italian government declared a state of emergency and stopped flights from and to China. Apart from two Chinese tourists who were promptly isolated, there was no confirmed case until February 21 when a patient with anomalous pneumonia was diagnosed with COVID-19 in Codogno, Lombardy. Shortly after that, new cases were discovered in other towns in Lombardy and Veneto. On February 22, a decree imposed the quarantine of more than 50,000 people from 11 municipalities (comuni) in Northern Italy (so called zone rosse). In other areas of Emilia-Romagna, Lombardy, and Veneto (zone gialle), schools, theaters, clubs, and cinemas were closed and social and sports events were suspended. On March 4, all schools and universities across Italy were closed for two weeks and all sporting events could be played only behind closed doors until April 3. As the outbreaks continued and the number of deaths soared, on March 8, all 12 provinces in Lombardy and 14 provinces in Piedmont, Emilia-Romagna, Veneto, and Marche, were put under lockdown. Two days later, the lockdown was extended to the whole country. Steep penalties were announced for violators, including the possibility of three months of imprisonment. On March 11, the government prohibited almost all commercial activities except for supermarkets and pharmacies. On March 21, all nonessential businesses and industries were closed, and movement of people was restricted. In May, many restrictions were progressively eased. Freedom of movement across regions and other European countries was restored on June 3.

\subsection{Portugal}

The first cases of COVID-19 in Portugal were recorded on March 2. On March 18, the entire Portuguese territory entered in a State of Emergency, which lasted until May 2. During the Easter week (April 9 to 13), the government decreed special measures to restrict people movements between municipalities (concelhos) with few exceptions, closing all airports to civil transportation. On May 4, restrictions started to be eased and small stores reopened. On May 18, nurseries, the last two years of the secondary school, restaurants, cafes, medium-sized stores, and some museums reopened. 


\subsection{Spain}

The first case of a patient with COVID-19 in Spain was a foreign tourist on January 31. Broader diffusion began by mid-February and all 50 provinces had confirmed cases by mid-March. A lockdown was imposed on March 14. Starting March 30, all nonessential workers were ordered to remain at home for the next two weeks. COVID-19 spread rapidly and by March 25, the official death toll in Spain surpassed that of China with most cases concentrated in Madrid. The number of deaths peaked in early April and progressively declined until June 1 which was the first day without COVID-19 related deaths. The first local lockdown was announced on March 7 for a small municipality. On March 12, the lockdown was extended to four municipalities in Catalunya with 70,000 people affected. On March 14, the entire country entered in the state of emergency and many nonessential activities were closed, such as restaurants and museums. Citizens were still permitted to travel to work and buy essential items. The authorities in some autonomous communities, including the Basque Country, Murcia, Balearic Islands, Catalunya, announced additional emergency measures. On March 28, all nonessential workers were ordered to stay home from March 30 to April 9. Progressive easing of the lockdown started at the beginning of May. On May 11, the opening of small shops, of terraces at half capacity, and of places of worship at one-third capacity was allowed in 26 provinces and territories comprising about half of the population.

\section{A Unique Dataset}

We use anonymized and aggregated data on mobility provided by Vodafone through a confidential agreement. By analyzing connections of mobile phones to cell towers, Vodafone can create mobility indexes differentiated across gender and age groups using the information that customers provide when signing up for postpaid contracts. ${ }^{1}$ The age groups include four categories: people aged between 18 and 24, between 25 and 44, between 45 and 64, and 65 and above. ${ }^{2}$ For Spain and Portugal, however, some daily observations are missing, especially for the oldest and youngest age groups. In these cases, we linearly interpolate the series to have a balanced panel throughout the sample period.

\footnotetext{
1 These indicators were prepared by Vodafone's Big Data and Artificial Intelligence team. To protect the privacy of individuals and minority groups, the data have been provided in anonymized form, reporting the average mobility for a given gender and age group at the provincial level when a minimum of 50 customers are available. Furthermore, the data sharing protocol was subject to technical and organizational controls including an ethical assessment of the analysis prior to its implementation.

${ }^{2}$ In a few cases, the age information is inferred. For example, in Spain, the age group 18-24 is separated from family contracts based on several factors, including the amount of data used. Furthermore, in Portugal customers' age is based on the sequential number of personal identification cards that allow to infer people's age with an error of five years at most.
} 
(a) Italy

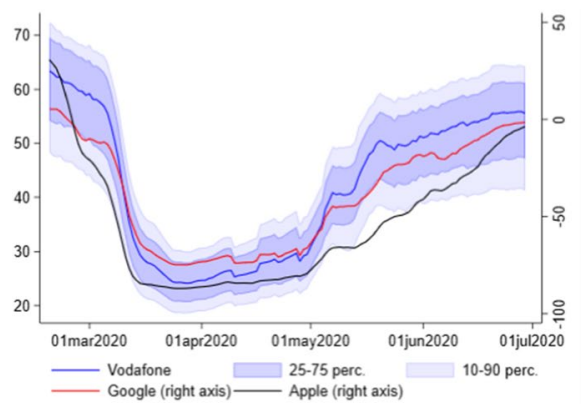

(b) Portugal

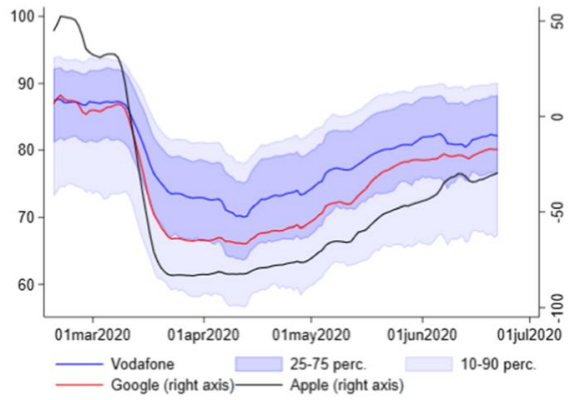

(c) Spain

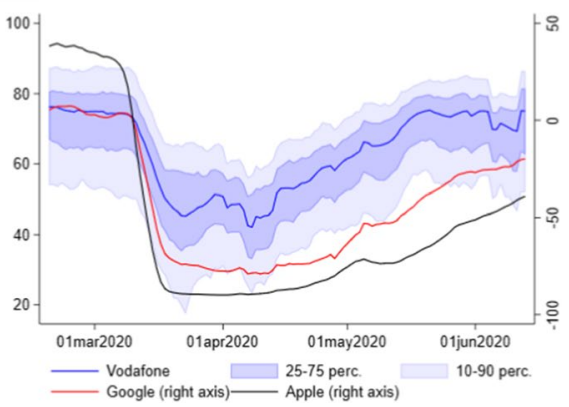

Fig. 1 Mobility Levels from Apple, Google, and Vodafone. Notes: The lines denote the country-level mobility levels. In the case of Vodafone, the line corresponds to the cross-province population-weighted average of the percent of people moving, using 2018 population levels as weights; and the shaded areas denote the cross-province interquartile range (dark blue) and the cross-province interdecile range (light blue). In the case of Google, the line corresponds to the average of country-level mobility indicators at retail, grocery, parks, transit, and workplace locations, where mobility is defined relative to pre-crisis levels. And in the case of Apple, the line denotes the country-level indicator of mobility, which is computed from the number of requests made to Apple Maps for directions. (Color figure online)

The mobility indicator used in the analysis captures the percentage of people in a given province and demographic group that leaves home in a day. ${ }^{3}$ The home location of each customer is identified by monitoring cell connections during the night. The top 3 cells that a phone connects to between $10 \mathrm{pm}$ and $5 \mathrm{am}$ are considered as home cells. A customer is recorded as leaving home if the phone connects to a cell different from the home cells. More details on the data construction are provided in Lourenco et al. (2020).

The mobility patterns detected by Vodafone are broadly in line with those according to Apple and Google data. ${ }^{4}$ Figure 1 shows that all indicators correlate fairly

\footnotetext{
${ }^{3}$ The data are disaggregated geographical at the NUTS-3 provincial level as defined by Eurostat to standardize data collection across the economic territory of the European Union.

${ }^{4}$ Apple mobility data are available at https://covid19.apple.com/mobility, and Google mobility data are available at https://www.google.com/covid19/mobility.
} 
(a) Women-men differential

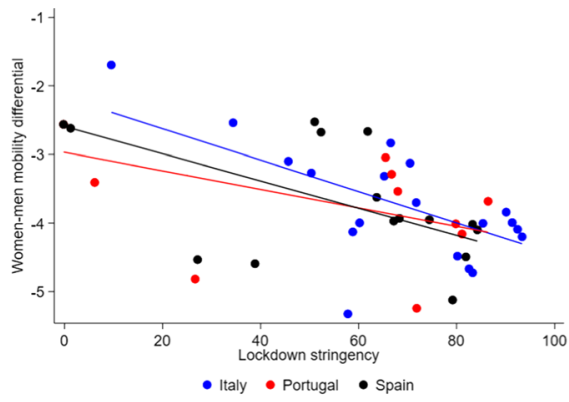

(c) $25-44$, differential with $65+$

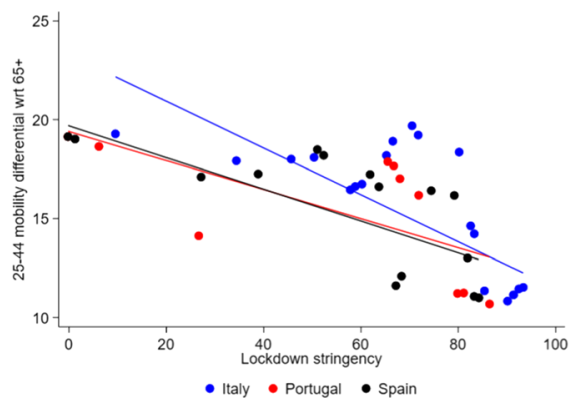

(b) 18-24, differential with $65+$

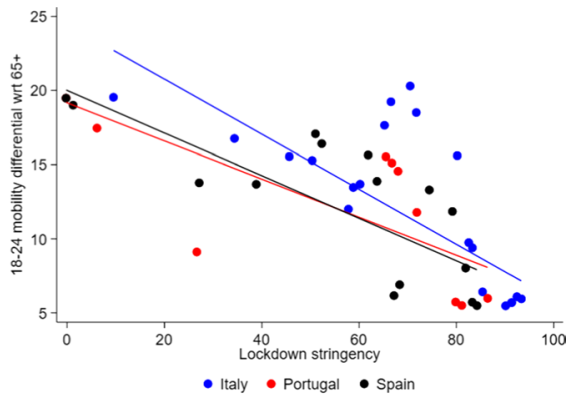

(d) $45-64$, differential with $65+$

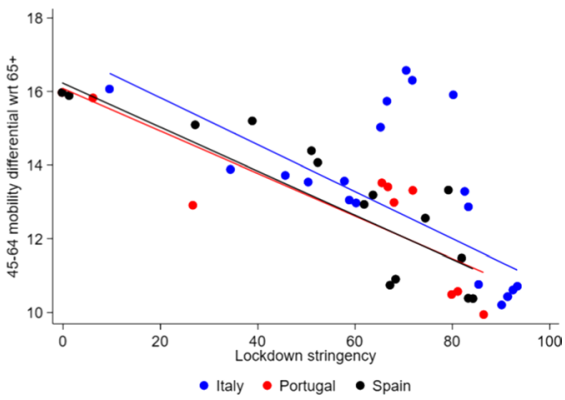

Fig. 2 Mobility and Lockdown Stringency. Notes: Panel (a) presents a binned scatter plot showing the mobility differential between women and men over the stringency of lockdowns during the period of analysis. Panels (b) to (d) present similar scatter plots but considering the differential in percentage points between people in different age groups relative to people aged 65 and above. Each dot denotes the cross-province average at any given time. The mobility indicator is residualized with respect to days of the week fixed effects. The lines denote the linear fit

closely at the national level. Correlations between the Vodafone indicator and the Apple and Google indicators range between 93 and 99 percent for Italy and Portugal and are 72 and 88 percent for Spain. The geographical disaggregation of the Vodafone data allows to appreciate the heterogeneity across provinces. In all the three countries, the interdecile range of the mobility indicator is as large as 20 percentage points. Yet, such dispersion remains broadly constant over time.

The key advantage provided by the Vodafone data is the ability to differentiate mobility across gender and age groups. This makes it possible to examine whether lockdowns have heterogeneous effects on people's mobility depending on gender and age. Figure 2 provides preliminary evidence in this regard. Panel 2 a shows for each country the correlation between the stringency of lockdowns during the period of analysis and the average mobility differential between women and men. In all countries, lockdowns have been associated with a larger drop in women' mobility relative to men's. The other three panels in Figure 2 show the correlation between the stringency of lockdowns and the mobility differential relative to the oldest age category of 65 and above. The charts suggest that lockdowns reduce more strongly 
the mobility of working age people relative to people aged 65 and above. The rest of the analysis will test more formally for these patterns using RD approaches and local projections.

\section{Heterogeneous Effects on Mobility Across Gender}

In this section, we examine whether lockdowns have a different effect on the mobility of women and men. Assessing the impact of lockdowns on mobility is a challenging task because the decision to deploy lockdowns is not random. For example, governments are more likely to impose lockdowns when health risks become more acute. At that time, people voluntarily reduce social interactions because they fear being infected or infecting others. If voluntary social distancing is not controlled for, the empirical analysis would thus overplay the impact of lockdowns on mobility. Policymakers may also impose lockdowns when mobility is too high, thus leading to a spurious association between lockdowns and high mobility.

To alleviate these endogeneity concerns, we use two empirical strategies. First, we employ RD designs that focus on high-frequency changes in mobility around specific lockdown measures, thus reducing the risk that other factors may affect mobility at the same time. Second, we use local projections that control for lagged mobility and for the severity of the country's epidemic based on the number of new infections. To further strengthen identification, local projections are estimated using data from provinces that did not experience severe outbreaks and thus for which the adoption of national lockdowns was mostly an exogenous event. The use of local projections is also helpful to examine how people voluntarily reduce mobility in response to rising infections.

\subsection{Regression Discontinuity}

To test whether lockdowns have unequal effects across gender, we first use an RD approach in a similar spirit to Davis (2008), Anderson (2014), and Chetty et al. (2020). With respect to a standard cross-sectional RD setting, in this case the running variable is time and the treatment date is a particular temporal threshold, making this approach akin to an event study exercise. As in more standard RD, endogeneity is addressed by considering a narrow bandwidth (in this case a time window) around the introduction of the treatment. The identification assumption is that, within this interval, unobserved confounding factors affecting the outcome variable are likely to be similar. In our context, this means that no other factors affecting mobility should change close to national stay-at-home orders. ${ }^{5}$

\footnotetext{
5 Of course, the number of contagions surged in the weeks leading to the stay-at-home orders, which were imposed because of this surge. However, the identifying assumption is that there was no discontinuity in the number of cases in the day of the orders. To test if this assumption is valid, we did an RD on cases at the time of lockdowns. There is no clear discontinuity.
} 
Fig. 3 Impact of Stay-at-Home Orders on Mobility, by Gender (Age group 25-44, percentage of people moving). Notes: The chart shows the percentage of men and women moving divided into 20 equal-sized bins. The series are residualized with respect to province and day-ofthe-week fixed effects

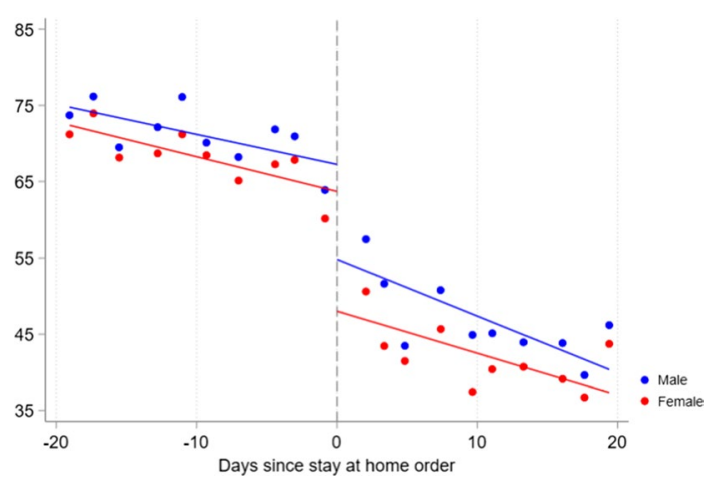

Figure 3 uses a bin scatter plot to present preliminary evidence that lockdowns are associated with a discontinuity in the mobility of women relative to men. Each dot represents the average mobility levels of men and women calculated using 20 equally sized bins around the introduction of national stay-at-home orders. We start by considering people aged between 25-44 for whom the differential effect of lockdowns across gender is the greatest, as we will later show. Mobility data are residualized with respect to province and day-of-the week fixed effects. The figure shows that the introduction of stay-at-home orders led to a sharp drop in the mobility of both men and women. The percentage of people living their homes in a day declined by about 15 points. Yet, the impact on women was stronger, as their mobility declined by about 3 percentage points more than for men.

In most provinces, the adoption of stay-at-home orders coincided with or rapidly followed the decision to close schools. Therefore, the gender gap in Fig. 3 could be driven by women carrying a disproportionate burden in caring for children when they are at home. To shed light on this aspect, we take advantage of the fact that five regions in Northern Italy closed schools well in advance of the national stayat-home order. ${ }^{6}$ Using mobility data from provinces in Northern Italy, Fig. 4a presents an RD exercise with two discontinuities: the first is set on February 23, the day when local schools closed, and the second one on March 9, when the national lockdown was implemented. The divergence in mobility between men and women started already at the time of school closures. Men's mobility declined very marginally when schools closed, while women's mobility saw a clear discontinuity. This corroborates the hypothesis that women carry uneven responsibilities in looking after the children when schools are closed.

Figure $4 \mathrm{~b}$ provides additional evidence about the importance of school closures by examining the mobility gender gap across all provinces in Northern Italy. The heat map reports the difference between the mobility of men and women through time, with darker colors representing a decline in women's mobility relative to

\footnotetext{
${ }^{6}$ Schools in Northern Italy closed on February 23. On March 4, the Italian government closed all schools and universities nationwide. The national stay-at-home order was announced with a Presidential Decree on March 9.
} 
(a) Average impact across provinces

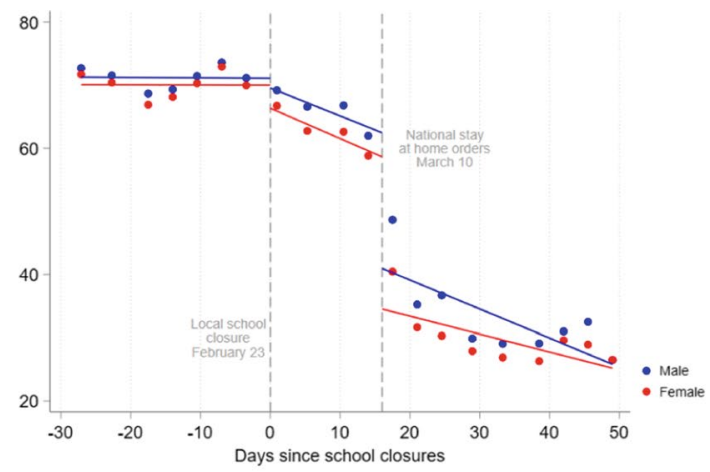

(b) Impact across each provinces

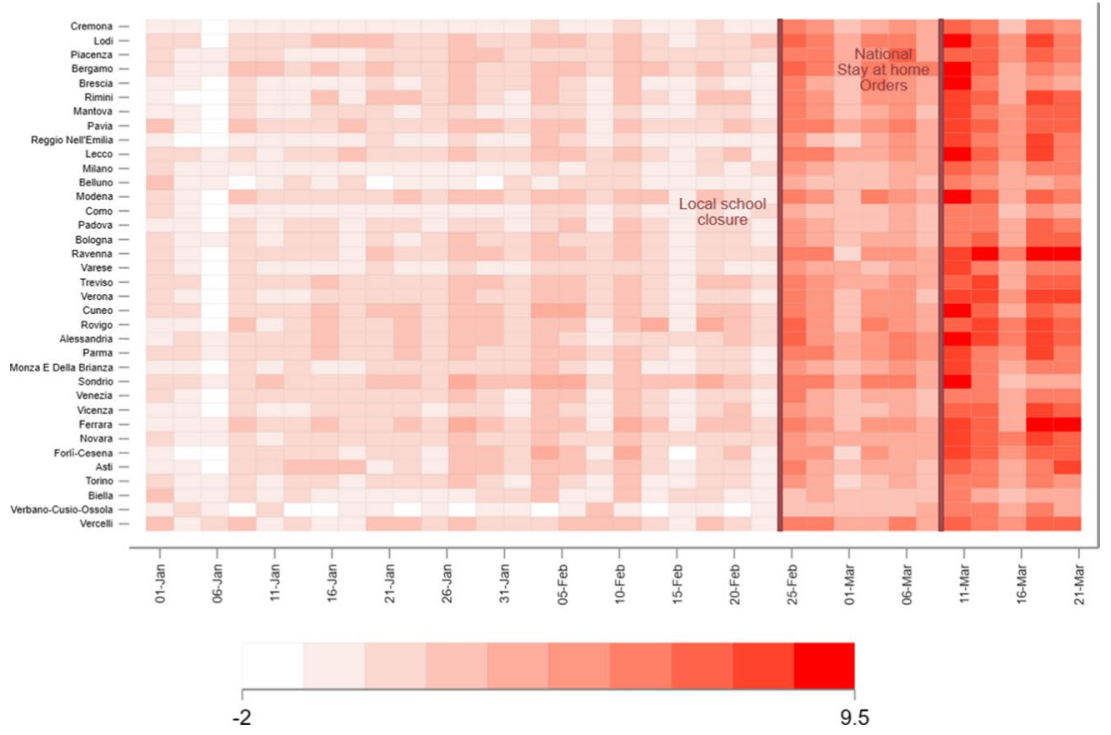

Fig. 4 Impact of School Closures and Stay-at-Home Orders in Northern Italy (Age group 25-44, percentage of people moving). Notes: Panel (a) reports a binned scatter plot where the percentage of people moving is divided into 20 equal-sized bins. The series are residualized with respect to province and dayof-the-week fixed effects. Panel (b) reports the difference between men and women mobility in FriuliVenezia-Giulia, Emilia-Romagna, Lombardy, Piemonte, and Veneto. Local school closures were introduced on February 23rd, and national stay-at-home orders on March 9th

men's. The mobility of men and women was similar before February 23. When schools closed, the heat map shows darker colors across all provinces, reflecting 
Table 1 RD Estimate of the Gender Gap by Age Group

18-24 25-44 $45-64 \quad 65+$

\begin{tabular}{lllll}
\hline Stay-at-home & $-19.60 * * *(0.59)$ & $-12.95^{* * *}(0.46)$ & $-12.52 * * *(0.41)$ & $-11.76^{* * *}(0.46)$ \\
$\begin{array}{l}\text { Women } \times \text { stay-at- } \\
\text { home }\end{array}$ & $-2.31 * * *(0.50)$ & $-3.24 * * *(0.35)$ & $-1.74 * * *(0.34)$ & $1.55^{* * *}(0.57)$ \\
Observations & 13,909 & 14,102 & 14,151 & 13,102 \\
$R$-squared & 0.87 & 0.86 & 0.87 & 0.82
\end{tabular}

Notes: The table reports the coefficient on the stay-at-home variable and the coefficient on the interaction between the gender dummy and the stay at home variable. All regressions include the gender dummy, a variable for the number of days since the introduction of the stay-at-home order, the interaction terms of the latter with the stay-at-home variable and with the gender dummy, and province and day-of-the-week fixed effects. Standard errors are clustered at the province level. $* * * p<0.01, * * p<0.05, * p<0.1$

a disproportionate reduction in women's mobility. The adoption of stay-at-home orders led to a further widening of the mobility gap. ${ }^{7}$

The differences in drop in mobility across age groups further confirm that school closures impacted disproportionately women's mobility. The analysis presented so far has focused on people aged between 25 and 44 . These cohorts are more likely to have young kids that require home supervision when schools are closed. ${ }^{8}$ A natural test of our conjecture is to test if the mobility gap was different for other age ranges.

To analyze the impact of lockdowns on the gender gap across all age groups and to test for the statistical significance of the discontinuity at the time of the lockdown, we follow Anderson (2014) and estimate this local linear regression:

$$
\begin{aligned}
\operatorname{mob}_{i, g, a, t}= & \alpha_{p}+\tau_{\text {dow }}+\beta \text { stay }_{j, t}+\phi \text { women }_{i}+\gamma \text { date }_{j, t}+\theta \text { stay }_{j, t} \times \text { date }_{j, t} \\
& +\lambda \text { women }_{i, a} \times \operatorname{stay}_{j, t}+\text { date }_{j, t} \times \text { women }_{i}+\mu \text { stay }_{j, t} \times \text { date }_{j, t} \times \text { women }_{i}+\varepsilon_{i, g, a, t}
\end{aligned}
$$

where $\mathrm{mob}_{i, g, a, t}$ is the mobility indicator provided by Vodafone capturing the percentage of people moving in province $i$, of gender $g$ and age group $a=\{[18,24] ;[25,44] ;[45,64] ;[65+]\}$, at time $t$; stay $_{j, t}$ is the treatment variable for country $j$ (with $i \in j$ ), equal to one when the national stay-at-home orders are in place; women $_{i, a}$ is a dummy variable equal to one when the dependent variable refers to the mobility of women; date ${ }_{j, t}$ is the number of days since the introduction of the stay-at-home order; and $\alpha_{p}$ and $\tau_{\text {dow }}$ are province and day-of-the-week fixed effects. The term date ${ }_{j, t} \times$ women $_{i}$ captures different trends for women and men (pre- and post-lockdowns). The coefficient $\beta$ captures the effect of the stay-at-home

\footnotetext{
7 Figure 4b provides also an additional insight. The provinces in Fig. 4(b) are listed in order of decreasing frequency of COVID-19 cases as a share of the province population on March 9. The absence of a clear vertical pattern indicates that the effects of school closures and lockdowns on the difference in mobility across gender were not correlated with the local intensity of the COVID-19 crisis.

8 In Italy, as in other countries, grandparents often play an important role in taking care of the kids while parents work. However, because COVID-19 affects disproportionately old people, social contacts between old and young people were discouraged. Therefore, the traditional arrangement was possibly less used, magnifying the effect of school closures on parents' mobility.
} 
orders on men's mobility, while $\lambda+\beta$ traces the effect on women's mobility. Standard errors are clustered at the province level. The identification assumption is that the term stay ${ }_{j, t} \times$ date $_{t}$ should absorb any smooth relationship between the date $e_{j, t}$ and the error term $\varepsilon_{i, g, a, t}$ in the days around the introduction of the lockdown (Anderson 2014). This means that no other factor affecting mobility should change close to the national stay-at-home orders. Consistent with Fig. 3, we estimate Eq. (1) using a relatively narrow window of 20 days around the adoption of stay-at-home orders since our identification strategy aims at estimating $\beta$ and $\lambda+\beta$ by considering the mobility drop close to the introduction of lockdowns.

Table 1 reports the results for the baseline model. Column (2) shows that the mobility of women aged 25-44 declined by 3 percentage points more than men's when stay-at-home orders were imposed which coincided in most provinces with school closures. Column (1) and (3) present the results of the same specification for the age groups 18-24 and 45-64: the gap between women and men mobility is still present, statistically significant, but smaller, equal to 2.3 and 1.7 percentage points respectively. Finally, Column (4) shows that lockdowns no longer have a disproportionate effect on the mobility of women in the age group 65+. These results show that the mobility gender gap is the largest for people aged 25-44 that are more likely to have young children. Therefore, they suggest that childcare needs largely explain the disproportionate impact of lockdowns on women's mobility.

\subsection{Local Projections}

The RD approach used in the previous section shows that the adoption of specific lockdown measures reduced the mobility of women more forcefully. We now check if similar results hold when using local projections that exploit the entire variation in the stringency of lockdowns over the period of analysis.

To alleviate endogeneity concerns about lockdowns - namely that they are more likely imposed when the epidemic is worsening and when mobility is too high - the local projections control for the number of COVID-19 infections and for lagged mobility levels to capture pre-existing trends. Furthermore, we rely on an identification strategy that takes advantage of the disaggregation of the Vodafone mobility data at the NUTS-3 level. Italy, Portugal, and Spain imposed lockdowns on a national scale in reaction to localized outbreaks. For example, in Italy the government imposed a national lockdown in early March even though most of the infections were concentrated in a few provinces in Lombardy. Therefore, the adoption of national lockdowns was largely exogenous to the conditions prevailing in provinces with relatively low infections. Leveraging on this observation, we exclude from the regression sample of each country the provinces that (i) registered the first 100 (cumulative) cases, (ii) had the highest number of COVID-19 cases by the end of June 2020, and (iii) that had more than five percent of the country's total confirmed cases when the lockdown stringency index reached its maximum. ${ }^{9}$ The regression

\footnotetext{
9 These criteria lead to the exclusion of Bergamo, Brescia, Lodi, Milan, Torino, and Rome in Italy; Barcelona and Madrid in Spain; and Área Metropolitana do Lisboa, Área Metropolitana do Porto, Cávado, and Região de Aveiro, Tâmega e Sousa in Portugal. Adding these areas back into the sample does not affect the results as shown in the robustness section.
} 
thus examines the mobility response in the regions less affected by the virus for which the national lockdown was an exogenous event triggered by conditions elsewhere in the country.

Formally, to assess the differential impact of lockdowns on women's mobility, we estimate the following local projection regressions (Jordà 2005) using data for a particular age group:

$$
\begin{aligned}
\operatorname{mob}_{i, g, a, t+h}= & \alpha_{i}^{h}+\kappa_{g}^{h}+\tau_{t}^{h}+\sum_{p=1}^{P} \rho_{p}^{h} \operatorname{mob}_{i, g, a, t-p}+\sum_{p=0}^{P} \delta_{p}^{h} \operatorname{lock}_{j, t-p}+\sum_{p=0}^{P} \beta_{p}^{h} \ln \Delta \operatorname{cases}_{i, t-p} \\
& +\operatorname{women}_{i, a} \times\left(\sum_{p=0}^{P} \gamma_{p}^{h} \operatorname{lock}_{j, t-p}+\sum_{p=0}^{P} \psi_{p}^{h} \ln \Delta \operatorname{cases}_{i, t-p}\right)+\varepsilon_{i, g, a, t+h}
\end{aligned}
$$

where variable $\mathrm{mob}_{i, g, a, t+h}$ denotes the percentage of people moving in province $i$, of gender $g$ and age $a$, at time $t+h$, with $h=\{1, \ldots, 20\}$ being the horizon; $\ln \Delta$ cases $_{i, t-p}$ is the log of daily COVID-19 cases, which is used to track the stage of the pandemic, with $p$ being the lag length (set to a week to control for the persistence of the variable); and lock $_{j, t-p}$ is an index measuring the stringency of lockdowns for country $j$ (with $i \in j$ ), which also enters the specification with $p$ lags to account for persistence. ${ }^{10}$ The specification also features lags of the dependent variable to control for pre-existing trends; province and gender fixed effects to capture time-invariant characteristics specific to provinces, men, and women; and time-fixed effects to control for those factors that are common to all provinces. Standard errors are clustered at the province level.

To uncover the differential impact of lockdowns on women, we include an interaction term between the lockdown stringency index and a gender dummy women w $_{i, a}$, which is equal to one when the dependent variable refers to the mobility of women. Thus, the coefficient $\delta_{0}^{h}$ isolates the impact of lockdowns on men's mobility and $\delta_{0}^{h}+\gamma_{0}^{h}$ the one on women's mobility. The regressions are estimated on a sample of 163 provinces in Italy, Spain, and Portugal between January 1 and June 29, 2020.

Figure 5 shows the impact of a full lockdown that includes all measures used during the pandemic - among which travel restrictions, school and business closures, and stay-at-home orders - on the mobility of men and women aged 24 to 45 . The responses in panel 5(a) show that a full lockdown leads to a very significant decline in mobility for both men and women. Mobility starts to decline when the lockdown

\footnotetext{
${ }^{10}$ We use the lockdown stringency index provided by the University of Oxford's Coronavirus Government Response Tracker. This index is a simple average of nine sub-indicators capturing school closures, workplace closures, cancellations of public events, gatherings restrictions, public transportation closures, stay-at-home requirements, restrictions on internal movement, controls on international traveling, and public information campaigns, both at the subnational and national level. Since we want to measure the impact of actual restrictions at the national level, we reconstruct the index excluding public information campaigns (as they aim to promote voluntary social distancing) and considering only measures that were adopted at the national level.
} 
(a) Men vs Women

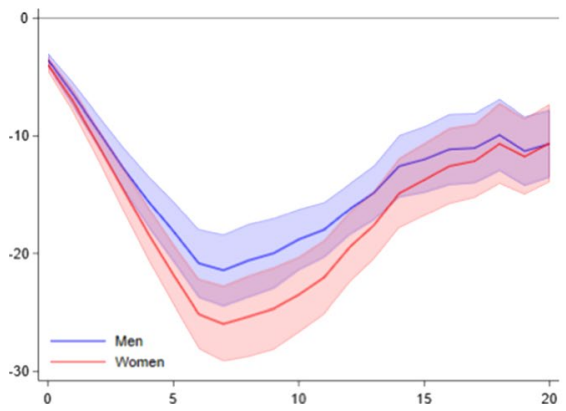

(b) Women-men differential

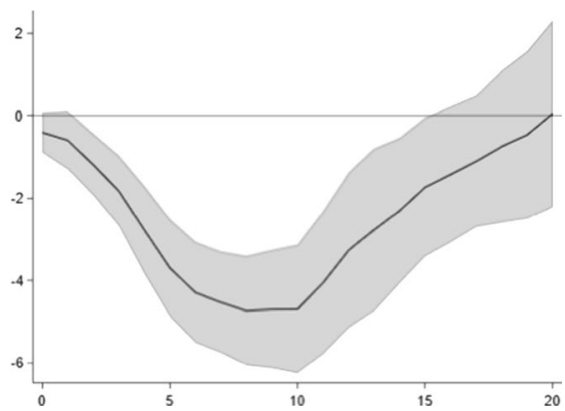

Fig. 5 Impact of a Full Lockdown on Mobility, by Gender (Age group 25-44, percentage of people moving). Notes: The $x$-axes denote the number of days, the lines denote the point estimates, and the shaded areas correspond to 90 percent confidence intervals computed with standard errors clustered at the province level

Table 2 Gender Gap at the Trough of the Estimated Response

\begin{tabular}{lllll}
\hline & $18-24$ & $25-44$ & $45-64$ & $65+$ \\
& $(1)$ & $(2)$ & $(3)$ & $(4)$ \\
\hline Lockdown stringency & $-27.07 * * *(1.80)$ & $-20.60 * * *(1.60)$ & $-19.75 * * *(1.57)$ & $-15.19 * * *(1.77)$ \\
Women $\times$ lockdown & $-3.58 * * *(0.95)$ & $-4.73 * * *(0.67)$ & $-3.69 * * *(0.73)$ & $2.33(1.46)$ \\
$\quad$ stringency & & & & \\
Days after the shock & 7 & 7 & 7 & 7 \\
Observations & 18,798 & 18,798 & 18,830 & 17,872 \\
Provinces & 163 & 163 & 163 & 157 \\
$R$-squared & 0.94 & 0.94 & 0.94 & 0.91 \\
\hline
\end{tabular}

Notes: The table reports the coefficient on lockdown stringency and the coefficient on the interaction term between the gender dummy and lockdown stringency at the trough of the estimated response. All regressions include the contemporaneous value and/or seven lags of the stringency index, the log of daily cases, the interaction between a gender dummy and the stringency index, and province, gender, and time fixed effects. Standard errors are clustered at the province level. $* * * p<0.01,{ }^{* *} p<0.05,{ }^{*} p<0.1$

is introduced, reaching the through after seven days. Mobility gradually resumes afterward as the lockdown stringency impulse dissipates, as shown in Fig. 15 of Appendix 1.

Most importantly, panel 5(a) reveals that lockdowns have an uneven effect on mobility across gender, impacting women more strongly. Women's mobility falls by 26 percentage points seven days after the introduction of lockdowns, while that of men declines by about 21 percentage points. Panel 5(b) shows that the differential between the mobility of women and that of men is statistically significant until the lockdown impulse weakens 15 days after the initial tightening.

These results corroborate the findings of the RD analysis that lockdowns tend to impact women disproportionately. The RD analysis also showed that the gender differential is the largest for people aged 25-44, probably because they are more likely 
(a) Men vs Women

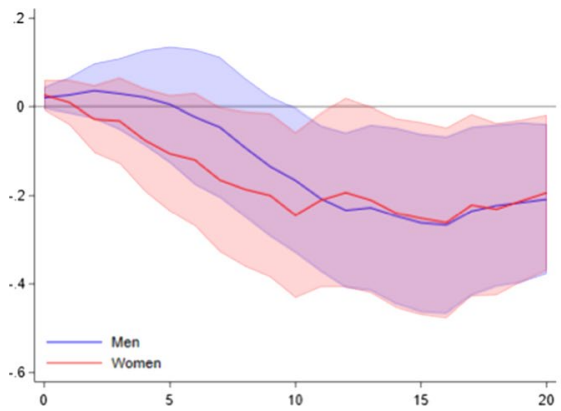

(b) Women-men differential

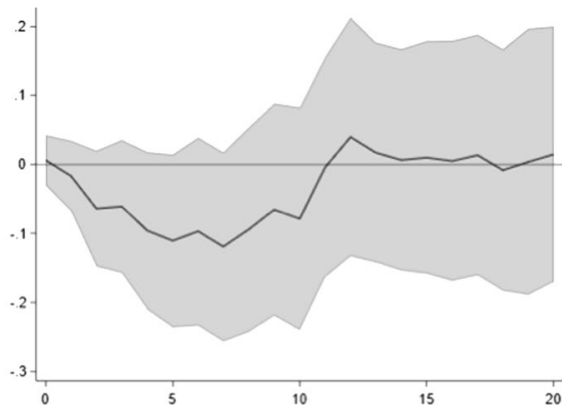

Fig. 6 Impact of a Doubling of COVID-19 Cases on Mobility, by Gender(Age group 25-44, percentage of people moving) .Notes: The $x$-axes denote the number of days, the lines denote the point estimates, and the shaded areas correspond to 90 percent confidence intervals computed with standard errors clustered at the province level

to have young children that have to be supervised at home when schools are closed. To check for the robustness of these findings, we re-estimate the local projections for the age groups $18-24,45-64$, and $65+$. Table 2 reports the largest mobility gap between women and men in response to a full lockdown over the 20-day horizon of the local projections. We confirm that women's mobility falls the most relative to men's for people aged 25-44, with a differential of 4.7 percentage points. The gender gap declines to 3.6 percentage points for people aged 18-24 and to 3.7 percentage points for those aged 45-64. For people in the age group 65+, the fall in women's mobility is statistically indistinguishable from the men's one. These results are thus closely aligned with those of the RD analysis.

Besides capturing the impact of lockdowns, the local projection in Eq. (3) also measures how mobility responds to an increase in COVID-19 infections holding constant the stringency of lockdowns. This is an important issue because during the pandemic people voluntarily reduced exposure to each other as they feared contracting the virus. For example, Aum et al. (2020), Goolsbee et al. (2020), and Maloney and Taskin (2020) document that mobility was tightly correlated with the spread of COVID-19 even after controlling for government lockdowns. In line with this literature, the specification in equation (2) sheds light on the strength of voluntary social distancing by capturing the response of mobility to rising COVID-19 infections for a given lockdown stringency. ${ }^{11}$ The interaction term between daily COVID-19

\footnotetext{
11 Besides reacting to the spread of COVID-19, people may opt to voluntarily self-distance also in response to other factors, such as public health announcements, news about celebrities being infected, or even the adoption of government lockdowns. As such, the analysis may underestimate the true extent of voluntary social distancing. Also, as shown by Adda (2016), higher mobility might lead to faster spread of viral diseases, generating some reverse causality between the outcome variables and COVID-19 infections. The dynamic structure of the estimation should alleviate this endogeneity concern.
} 
(a) Regression discontinuity

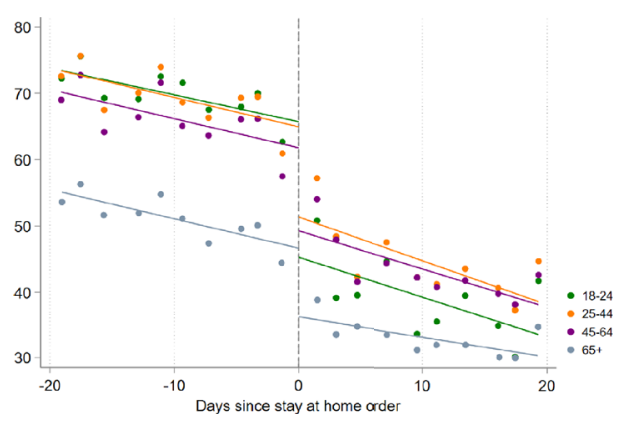

(b) Estimated impacts

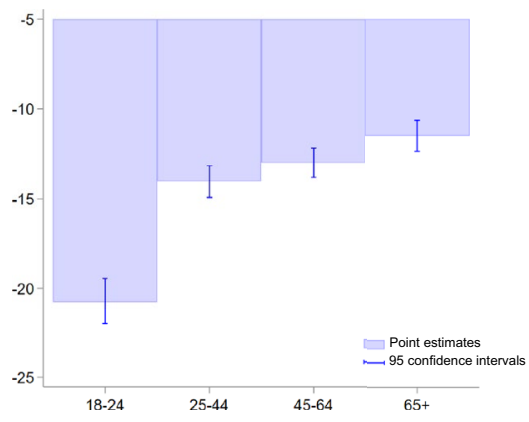

Fig. 7 Impact of a Stay-at-Home Orders on Mobility, by Age (Percentage of people moving) .Notes: Panel 7(a) presents a binned scatterplot where the people moving is divided in 20 equally sized bins. The series is residualized with respect to province, gender day-of-the-week fixed effects. Panel 7(b) reports the estimates of the percentage drop in people moving by age group. Standard errors are clustered at the province level. See also Table 5

infections and the gender dummy reveals if the extent of voluntary social distancing differs between men and women. Specifically, the coefficient $\beta_{0}^{h}$ measures the extent of voluntary social distancing for men, while the coefficients $\beta_{0}^{h}+\psi_{0}^{h}$ reflect the response of women.

Figure 6 shows how mobility responds to rising COVID-19 infections for a given lockdown stringency. An increase in COVID-19 cases has a negative effect on the mobility of both men and women. A doubling of daily COVID-19 cases leads to a contraction in mobility by about 0.3 percentage points 20 days after the introduction of the lockdowns. The effect is similar across men and women. Panel 6(b) shows indeed that there is no statistically difference across gender in how mobility responds to rising infections.

\section{Heterogeneous Effects on Mobility Across Age Groups}

In this section, we examine if lockdowns have a different impact on mobility depending on people's age. In line with the analysis on the effects across gender, we first examine the data using an RD approach. We then revisit the evidence using local projections which also allow us to examine if people respond differently to rising infections depending on their age.

\subsection{Regression Discontinuity}

We study the impact of stay-at-home orders on different age groups using the RD framework described in Sect. 4.1. Panel 7(a) shows graphical evidence of the impact of stay-at-home orders on the mobility of each age group. Each dot captures the 
average mobility of both women and men in a given age group from 20 days before to 20 days after the adoption of stay-at-home orders. We see that lockdowns drastically reduced people's mobility across all age groups.

Yet, the mobility drop was significantly stronger for younger cohorts. This is more clearly illustrated in panel 7(b) which shows the estimated mobility contraction for each age group using an RD specification akin to 1, with the associated 90 percent confidence intervals. The percentage of people below 25 years of age leaving home in a day declined by more than 20 points when stay-at-home orders were imposed. The mobility drop becomes progressively smaller for older people, being equal to only 11 percentage points for people aged 65 and above.

These results show that lockdowns tend to disproportionally impact the mobility of younger cohorts. This is not surprising if we consider that the mobility of people aged 65 and above - most of whom are retirees - was already significantly lower prior to lockdowns, as illustrated in panel 7(a). Younger people have instead to leave their homes on a daily basis to reach their work places and bring children to schools. Therefore, they are much more affected by lockdowns that, by impeding movements, have more adverse effects on their employment opportunities.

\subsection{Local Projections}

We now assess the impact of lockdowns across age groups using local projections based on the same identification strategy discussed in Sect. 4.2. Thus, the local projections control for the number of COVID-19 infections, lagged mobility, and are estimated using data from provinces that did not experience early and/or major outbreaks and thus for whom the adoption of national lockdowns was largely exogenous. Formally, we estimate the following specification:

$$
\begin{aligned}
\operatorname{mob}_{i, g, a, t+h}= & \alpha_{i}^{h}+\kappa_{a}^{h}+\tau_{t}^{h}+\sum_{p=1}^{P} \rho_{p}^{h} \operatorname{mob}_{i, g, a, t-p}+\sum_{p=0}^{P} \delta_{p}^{h} \operatorname{lock}_{j, t-p}+\sum_{p=0}^{P} \beta_{p}^{h} \ln \Delta \operatorname{cases}_{i, t-p} \\
& +\sum_{a=1}^{3} \operatorname{agegroup}_{i}^{a, g} \times\left(\sum_{p=0}^{P} \gamma_{p}^{s, h} \operatorname{lock}_{j, t-p}+\sum_{p=0}^{P} \psi_{p}^{s, h} \ln \Delta \operatorname{cases}_{i, t-p}\right)+\varepsilon_{i, g, a, t+h}
\end{aligned}
$$

The specification features interaction terms between the lockdown stringency index and age group dummies $\operatorname{agegroup}_{i, g}^{a}$, with $a=\{1=[18,24] ; 2=[25,44] ; 3=[45,64]\}$, where the excluded category is the age group $65+$. Hence, the impact of lockdowns on the mobility of people aged $65+$ at horizon $h$ is captured by $\delta_{0}^{h}$, while the impact on the other age groups $a$ is given by $\delta_{0}^{h}+\gamma_{0}^{a, h}$. The specification also includes interaction terms between COVID-19 infections and age groups to test whether the strength of voluntary social distancing differs across age. For a given level of lockdown stringency, the impact of rising COVID-19 cases on the mobility of people aged $65+$ is measured by $\beta_{0}^{h}$, and the one on other age groups by $\beta_{0}^{h}+\psi_{0}^{a, h}$. 
(a) Age groups

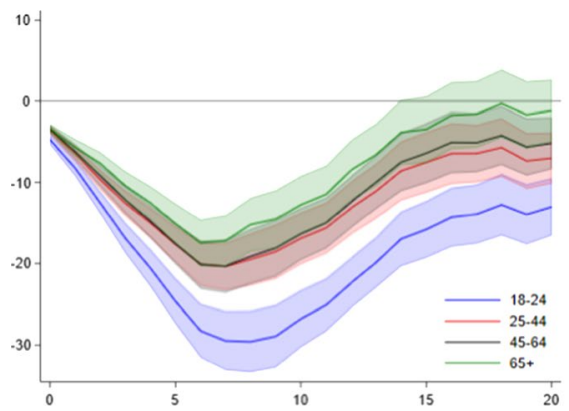

(b) Differential with 65+

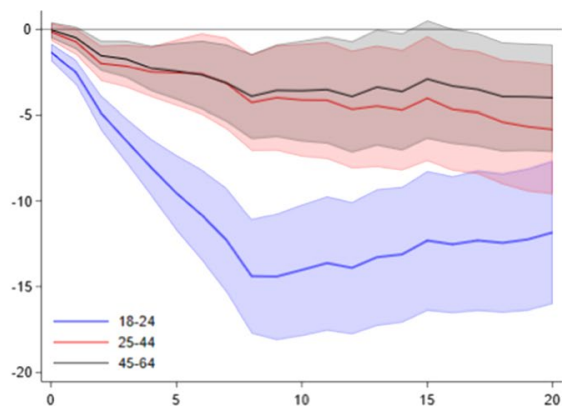

Fig. 8 Impact of a Full Lockdown on Mobility, by Age Group (Percentage of people moving). Notes: The $x$-axes denote the number of days, the lines denote the point estimates, and the shaded areas correspond to 90 percent confidence intervals computed with standard errors clustered at the province level

(a) Age groups

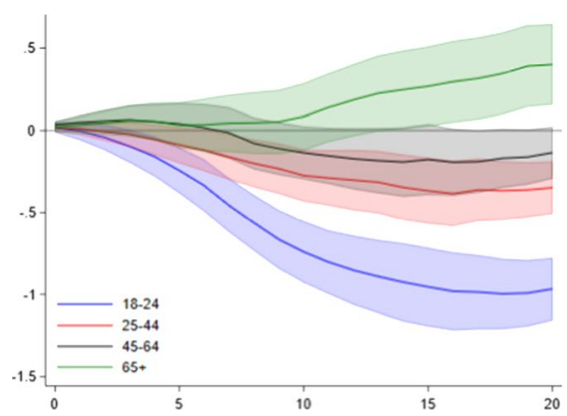

(b) Differential with respect to $65+$

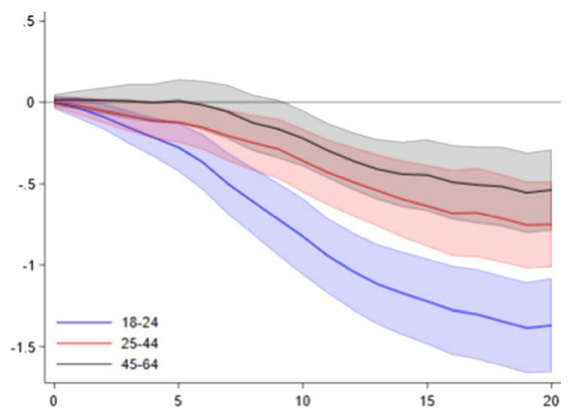

Fig. 9 Impact of a Doubling of COVID-19 Cases on Mobility, by Age Group(Percentage of people moving). Notes: The $x$-axes denote the number of days, the lines denote the point estimates, and the shaded areas correspond to 90 percent confidence intervals computed with standard errors clustered at the province level

Figure 8 shows the effects of lockdowns on the mobility of different age groups. As shown in panel 8(a), mobility declines sharply across all age categories during the first ten days after the introduction of a lockdown. Yet, the younger cohorts experience a considerably larger drop in mobility, reaching a trough of 30 percentage points nine days after the introduction of lockdowns for people aged 18-24. ${ }^{12}$

To illustrate the differences across age groups more clearly, panel 8(b) shows the mobility differential of each age group relative to people aged $65+$. The mobility differential of those aged 18-24 compared to people $65+$ is the largest and becomes

\footnotetext{
12 The mobility dynamics reflect the underlying impulse to the lockdown stringency that dissipates after two weeks as illustrated in Figure B1a of "Appendix 2."
} 
statistically significant since the beginning of the projection horizon. The drop in mobility for the age groups $25-44$ and 45-64 becomes statistically larger relative to people $65+$ a few days after the lockdown stringency impulse. These findings corroborate the results from the RD analysis in the previous section, showing that lockdowns impact the mobility of younger cohorts disproportionally.

The local projections also shed light on whether the extent of voluntary social distancing differs across age groups. Panel 9(a) shows that the youngest cohorts react most forcefully to a rise in infections. A doubling of COVID-19 cases leads to a fall in mobility by about one percentage point after 20 days for people aged 18-24. A rise in infections leads also to reduction in mobility for people aged 25-44 and 45-64, even though the effect is more modest. Mobility remains instead broadly unchanged for people aged $65+$, actually increasing marginally toward the end of the projection horizon. Panel 9(b) confirms that mobility declines more for all working-age groups relative to people $65+$.

These results are somewhat surprising because people aged $65+$ face much greater health risks from COVID-19 and should thus be more prone to isolate themselves when infections increase. Two considerations may explain our findings. First, the rise in infections reduces business activity in contact-intensive businesses, such as bars and restaurants, as people fear becoming infected. This in turn reduces employment in those sectors where many young people tend to work. Second, the larger response in the mobility of the young may reflect their stronger concerns about the virus. This is consistent with the evidence presented in Bordalo et al. (2020). Based on a survey of 1,500 Americans in May 2020, they find that perceptions about the health risks posed by COVID-19 decline sharply with age. The fact that younger generations seem more sensitive to the fear factor - measured as doubling COVID-19 cases - could also reflect that younger generations use more media and social media which emphasize the danger.

\section{Robustness}

In this section, we test the robustness of our results along several dimensions. We start by re-examining in Table 3 the impact of lockdowns on mobility across gender for peopled aged 25-44 based on the RD analysis. Column (1) reports the differential impact between women and men from the baseline specification in Sect. 4.1. It shows that lockdowns lead to a disproportionate decline in women's mobility. These results are based on a 20-day window before and after the adoption of stay-athome orders. In column (2), we show that similar results are obtained if we shrink the regression window to 10 days to further limit possible bias from unobservable confounders. The results are also robust in column (3) to excluding the regions in Northern Italy that introduced lockdown measures, such as school closures, before the national stay-at-home order, as shown in Fig. 4a, b. In columns (4) and (5) we exclude, one at a time, data for Portugal and Spain for which the mobility series are interpolated. The differential impact on women's mobility is confirmed on these different samples. Finally, in column (6) we control for the moving average of daily 


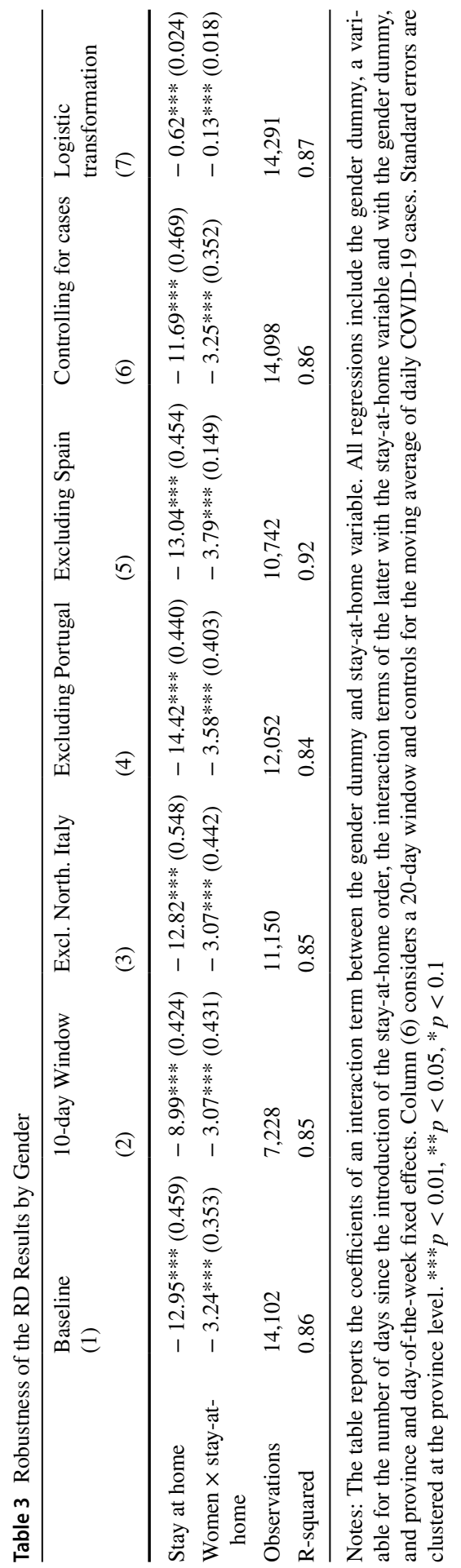




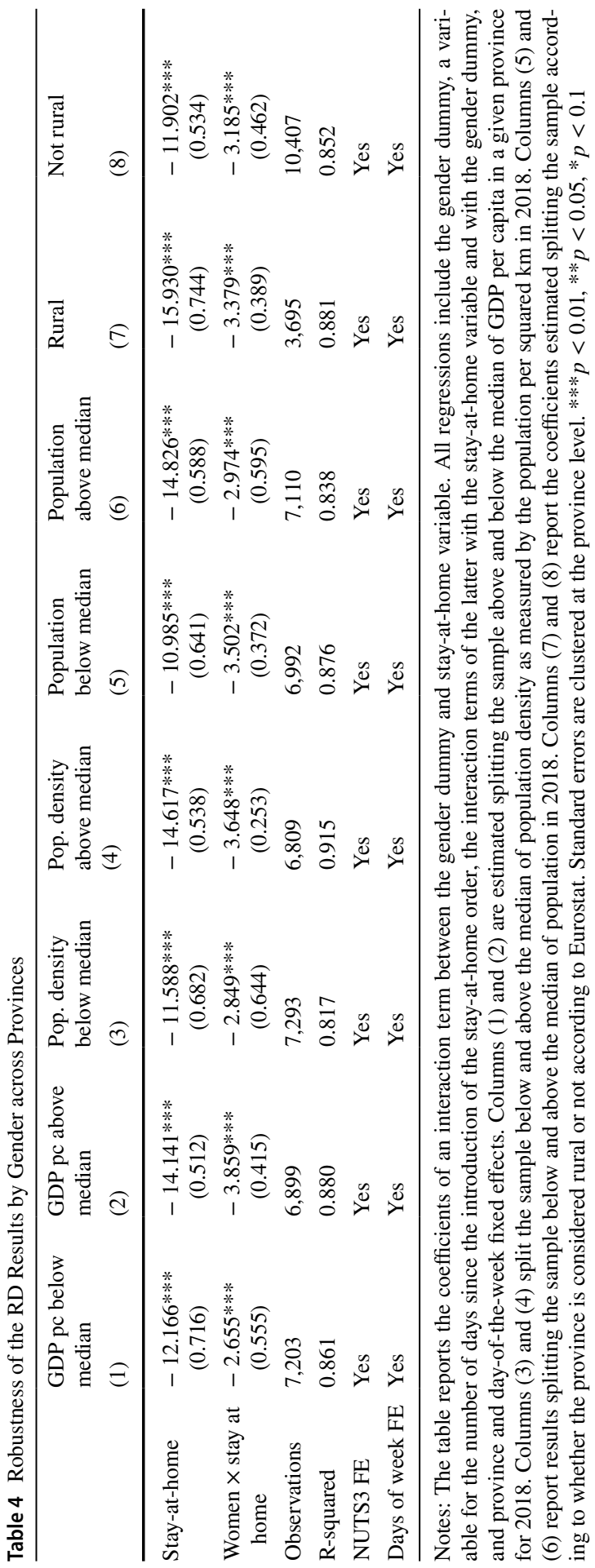




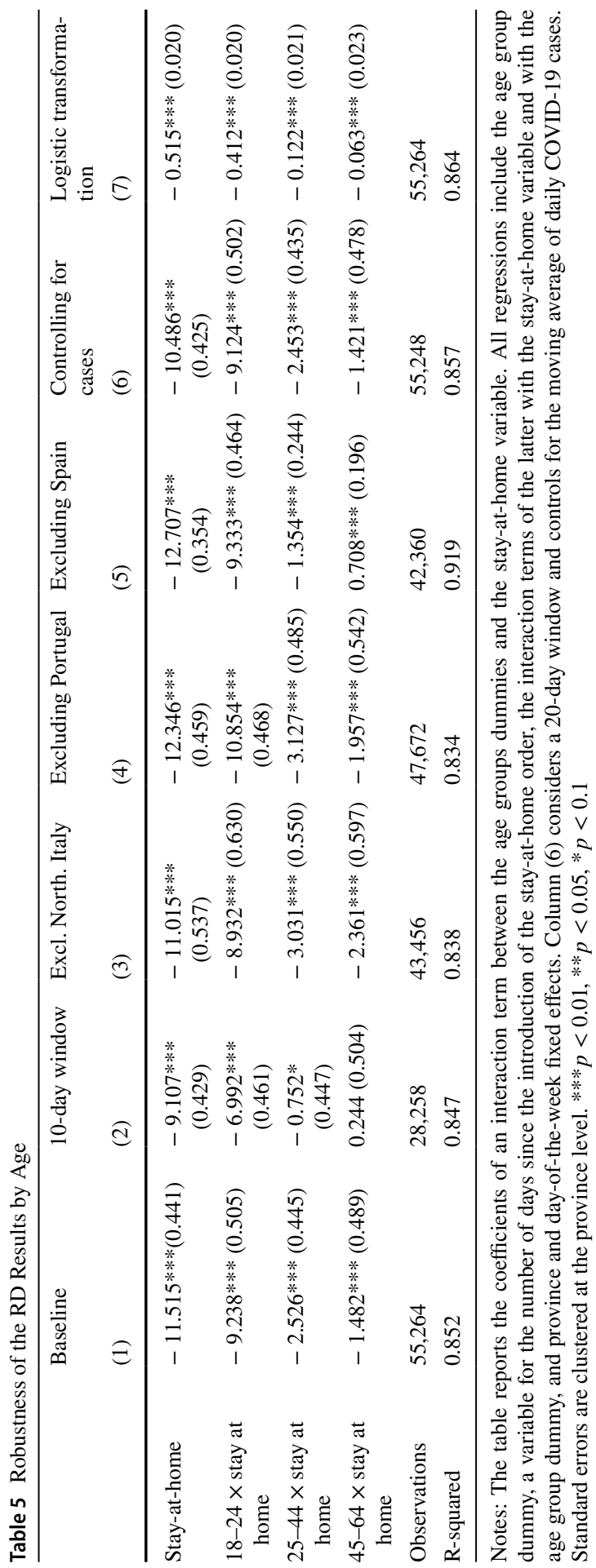




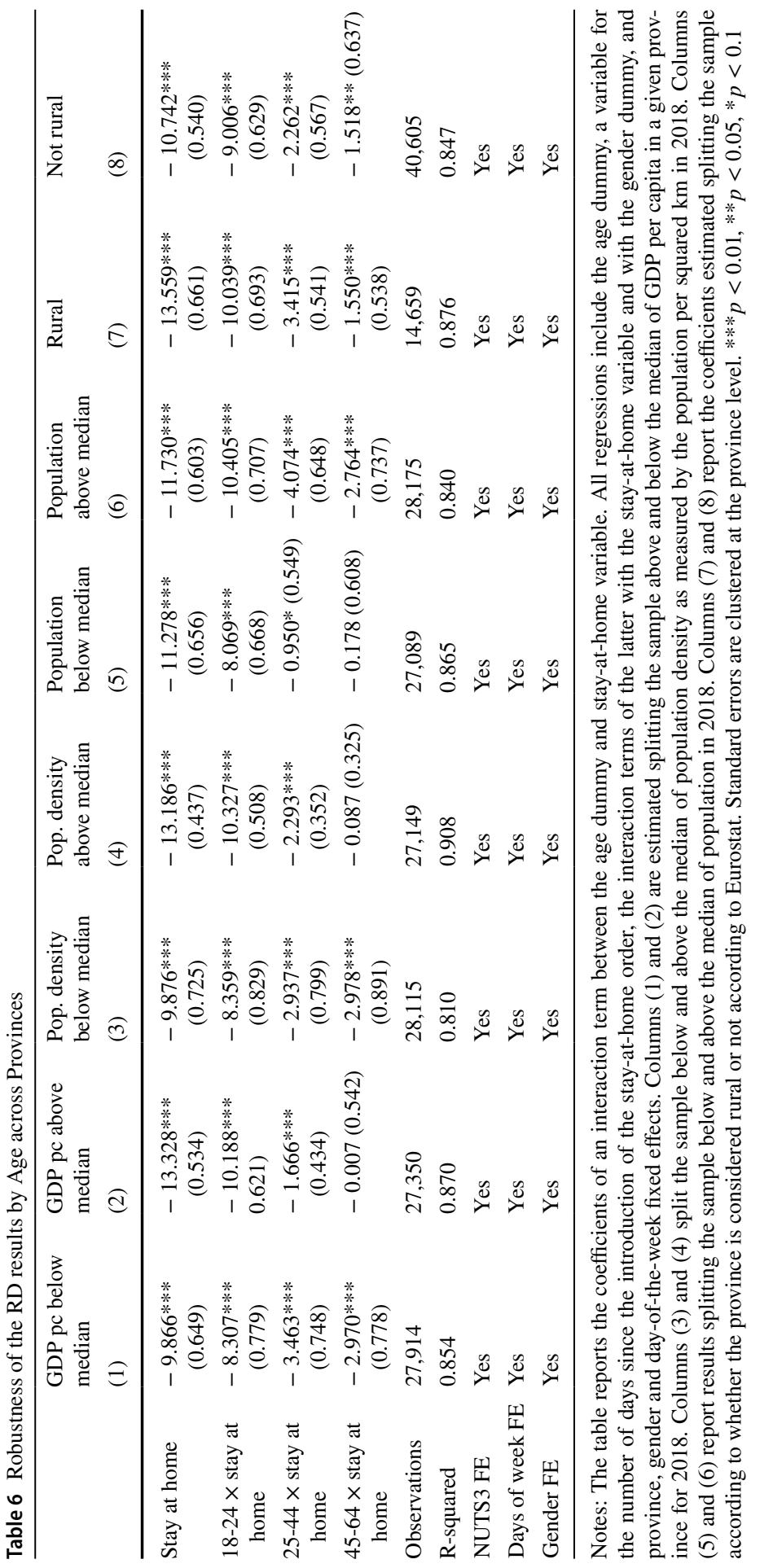


(a) Impact of a full lockdown, women-men differential

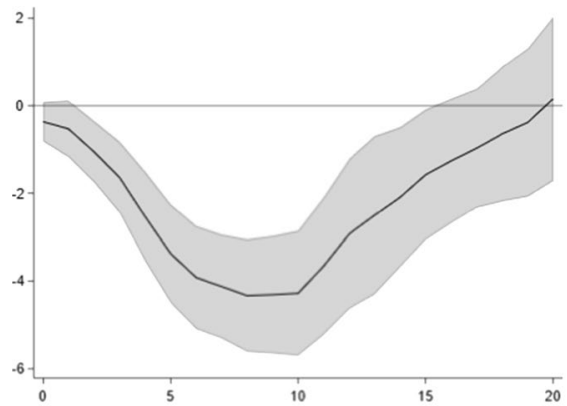

(c) Impact of a full lockdown, differential with respect to $65+$

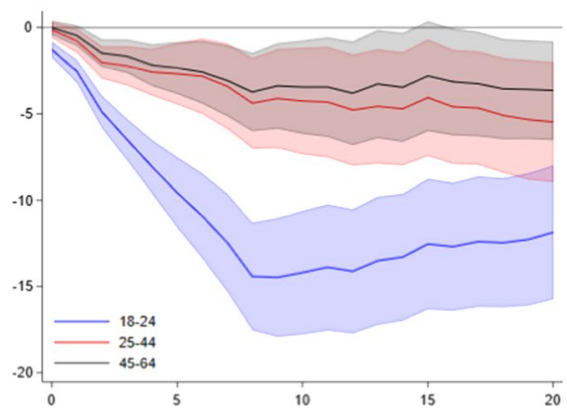

(b) Impact of a doubling of COVID-19 cases, women-men differential

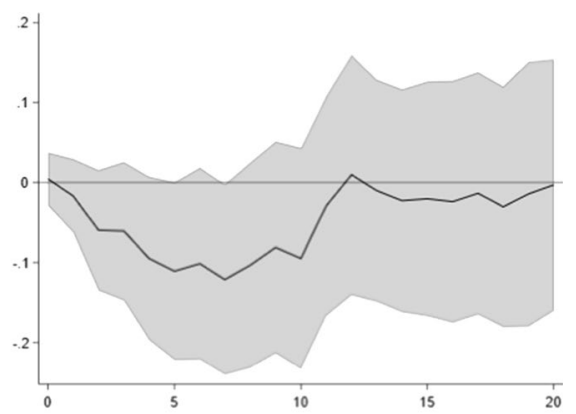

(d) Impact of a doubling of COVID-19 cases, differential with respect to $65+$

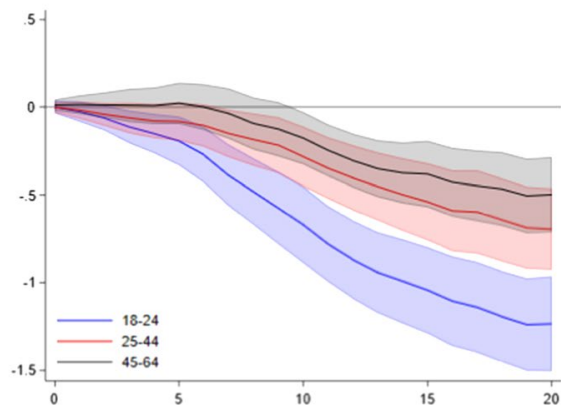

Fig. 10 Impact of Lockdowns and COVID-19 Cases using Full Sample (Percentage of people moving). Notes: The $x$-axes denote the number of days, the lines denote the point estimates, and the shaded areas correspond to 90 percent confidence intervals computed with standard errors clustered at the province level

COVID-19 at the province level to reduce concerns of omitted confounders that may have a discontinuous effect on the mobility. The inclusion of this control beyond province and day-of-the-week fixed effects does not alter the results. Finally, since the mobility indicators are bounded between 0 and 100 percent, we verify in column (7) that the results are robust to applying a logistic transformation.

We also verify if the results are robust across provinces with different characteristics. In Table 4, we rerun the RD analysis splitting the sample depending on whether provinces are above or below the median level of GDP per capita, population density, and population size. Furthermore, we split the sample based on whether provinces as considered rural or not by the Eurostat. Across all these samples, lockdowns continue to have a disproportionate effect on the mobility of women, thus confirming that the results are not driven by provinces with peculiar characteristics. Following a similar set of robustness tests, Tables 5 and 6 corroborate the RD findings that lockdowns have a stronger impact on the mobility of younger cohorts. 
(a) Impact of a full lockdown, women-men differential

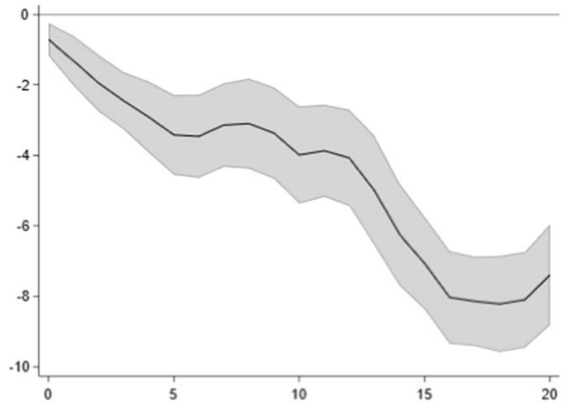

(c) Impact of a full lockdown, differential with respect to $65+$

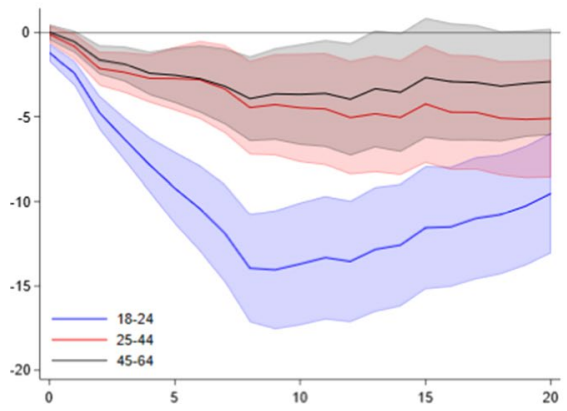

(b) Impact of a doubling of COVID-19 cases, women-men differential

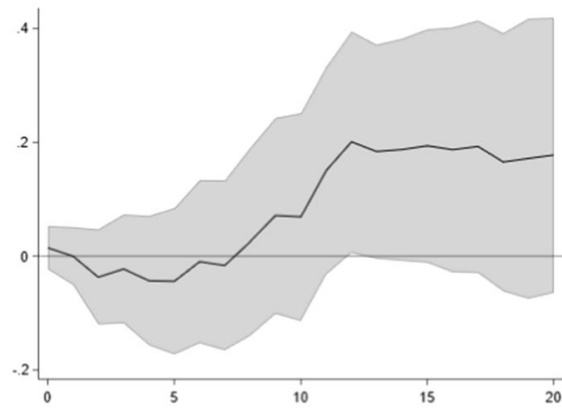

(d) Impact of a doubling of COVID-19 cases, differential with respect to $65+$

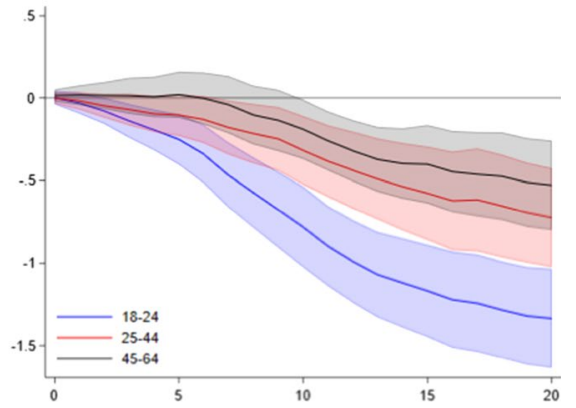

Fig. 11 Impact of Lockdowns and COVID-19 Cases using Day-of-the-Week Fixed Effects(Percentage of people moving). Notes: The $x$-axes denote the number of days, the lines denote the point estimates, and the shaded areas correspond to 90 percent confidence intervals computed with standard errors clustered at the province level

We also perform various robustness tests for findings of the local projections. As discussed in section 4.2, the local projections are estimated on a sample that excludes provinces with early and/or large outbreaks. While this approach mitigates endogeneity concerns regarding the introduction of lockdowns, it may affect our estimates if lockdowns or voluntary social distancing had different effects on mobility in regions more impacted by the virus. Thus, we test if our findings are robust to the inclusion of those provinces. Figure 10 shows the coefficients on the interaction terms that capture the differential impact of lockdowns and COVID-19 cases on mobility across gender and age groups. Panels 10(a) and 10(b) confirm that lockdowns hit women's mobility disproportionately and that voluntary social distancing was broadly similar across gender. Panels 10 (c) and $10(\mathrm{~d})$ also corroborate the baseline results that lockdowns and voluntary social distancing took a larger toll on 
(a) Impact of a full lockdown, women-men differential

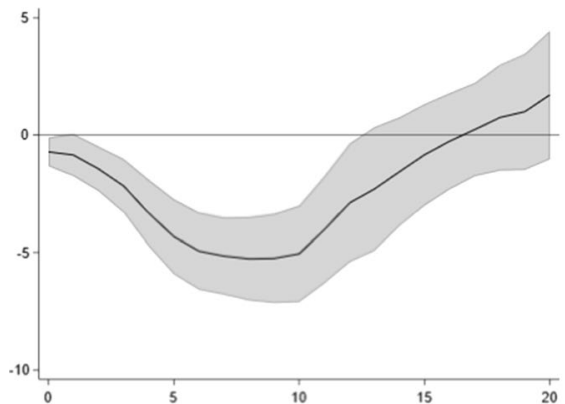

(c) Impact of a full lockdown, differential with respect to $65+$

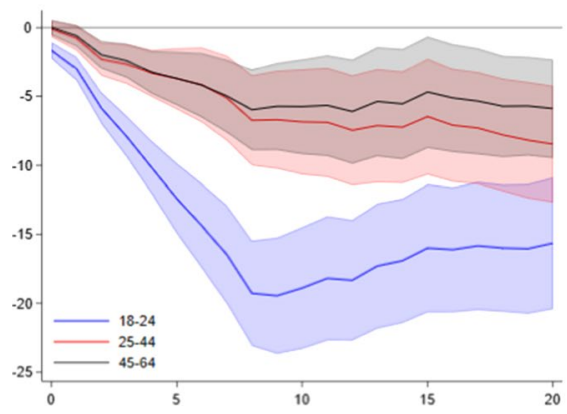

(b) Impact of a doubling of COVID-19 cases, women-men differential

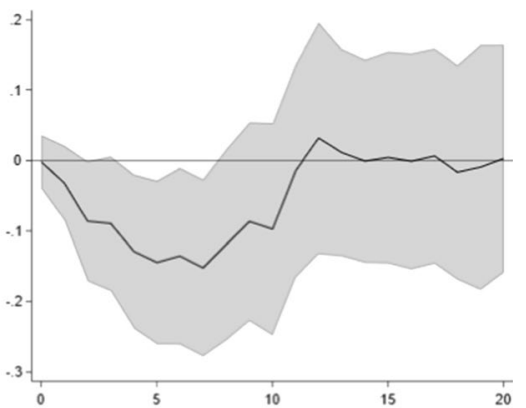

(d) Impact of a doubling of COVID-19 cases, differential with respect to $65+$

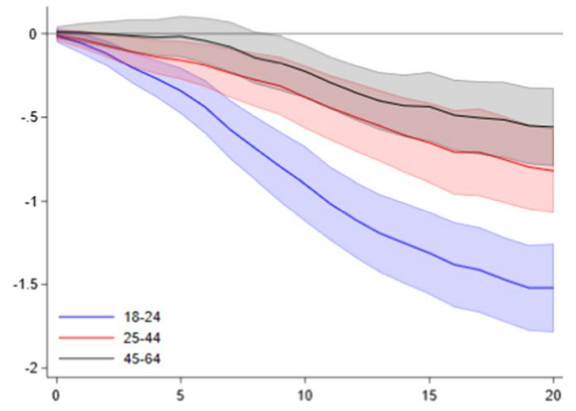

Fig. 12 Impact of Lockdowns and COVID-19 Cases Excluding Portugal (Percentage of people moving). Notes: The $x$-axes denote the number of days, the lines denote the point estimates, and the shaded areas correspond to 90 percent confidence intervals computed with standard errors clustered at the province level

younger cohorts. In terms of magnitudes, the estimated effects are virtually identical to those in the baseline.

We also examine if the results are robust to excluding time fixed effects which are used to capture movements in mobility that are common across provinces but are unrelated to the dynamics of lockdown stringency and COVID-19 infections. ${ }^{13}$ One could argue that controlling for them in the local projections may saturate the specification given the high-frequency of the data. We thus replace time fixed effects with day-of-the-week fixed effects. Panels 11(a) and 11(b) of Figure 11 confirm the disproportional impact of lockdowns on women's mobility compared to men's, as well as that rising infection do not generally have a statistically significant different effect across gender. If anything, the women-men mobility differential in response to lockdowns appear more persistent than with time fixed effects. Similarly, without

\footnotetext{
13 Such movements could be caused by public announcements by the government, public health officials, and international organizations, or by news about celebrities being infected.
} 
(a) Impact of a full lockdown, women-men differential

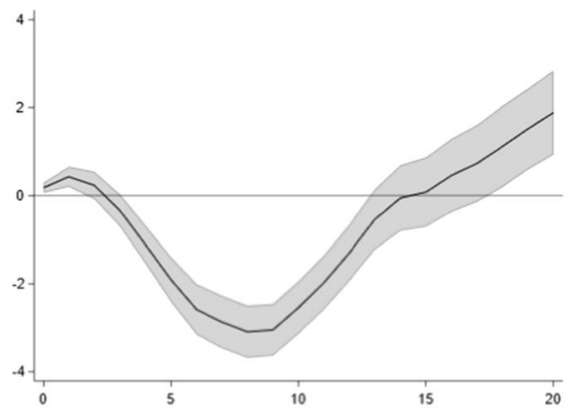

(c) Impact of a full lockdown, differential with respect to $65+$

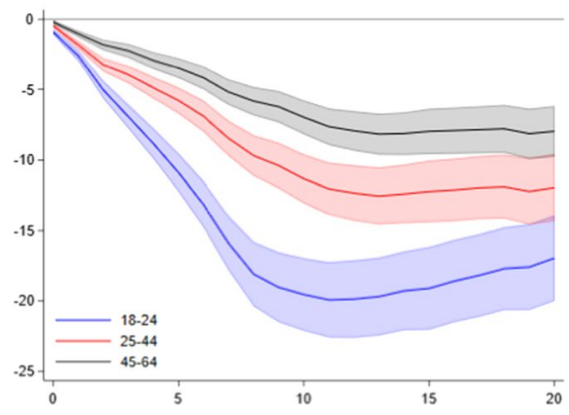

(b) Impact of a doubling of COVID-19 cases, women-men differential

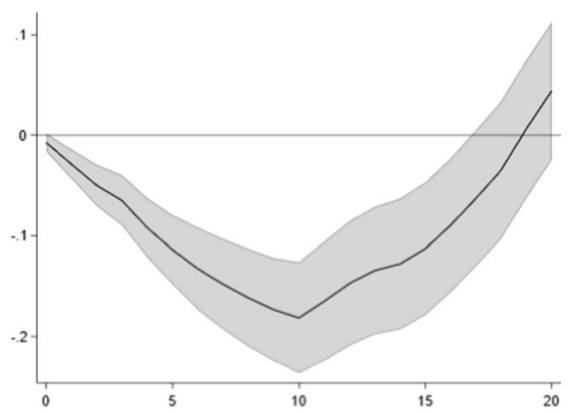

(d) Impact of a doubling of COVID-19 cases, differential with respect to $65+$

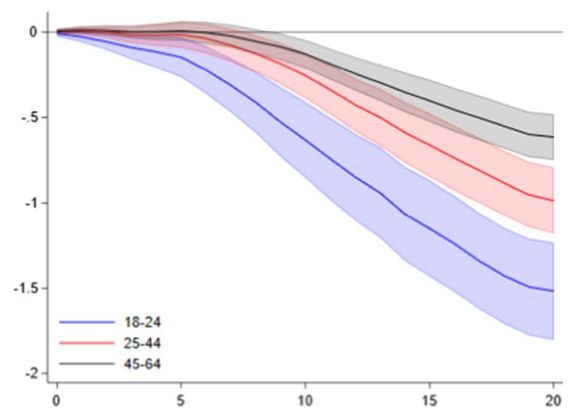

Fig. 13 Impact of Lockdowns and COVID-19 Cases Excluding Spain (Percentage of people moving). Notes: The $x$-axes denote the number of days, the lines denote the point estimates, and the shaded areas correspond to 90 percent confidence intervals computed with standard errors clustered at the province level

time fixed effects, panel 11(c) shows that lockdowns are still found to impact more strongly the mobility of younger people. Finally, panel 11(d) shows that the baseline findings about the impact of rising COVID-19 cases on the mobility of different age groups are robust to replacing time fixed effects with day-of-the-week fixed effects.

Another possible concern with the local projections is that the results could be affected by the linear interpolation of the missing observations for Portugal and Spain. Thus, we re-estimate the local projections excluding one of these two countries at a time. ${ }^{14}$ Figure 12 presents the results of the impact of lockdowns and voluntary social distancing when data for Portugal are excluded. Figure 13 repeats the exercise excluding Spain. In both cases, the results closely mimic those in the baseline analysis. The only difference is that an increase in COVID-19 cases tends to

\footnotetext{
14 Running regression with data for a single country would prevent the possibility of including time fixed effects.
} 
have a larger impact on women's mobility relative to men's, especially when Spain is excluded in panel 13(b). Yet, the point estimates are quantitatively very small.

As in the case of the RD analysis, we also verified that the results of the local projections are robust to applying a logistic transformation to the mobility indicator and to splitting the sample depending on province characteristics. In addition, to ensure that our findings do not depend on preexisting trends in mobility that are heterogeneous across gender or age groups, we include the interactions between lagged mobility and either the gender dummy or the age groups dummies. We find that our estimates are largely unchanged. Finally, we employ two different approaches to compute the standard errors which take into account the plausible correlation across NUTS-3 units: first, we cluster the standard errors at the NUTS-2 level (instead of NUTS-3); second, we follow the Driscoll and Kraay (1998) procedure which corrects for cross-sectional dependence. Both these approaches return similar results to the baseline ones. The results of these tests are available upon request.

\section{Conclusions}

We have used unique mobility indicators provided by Vodafone which differentiate by gender and age characteristics to shed light on several key themes that have emerged during the COVID-19 pandemic.

First, the analysis provides novel evidence about the disproportionate impact of the crisis on women. Lockdown measures reduce the mobility of women more than men's. This seems largely due to women carrying an uneven burden in caring for children when schools are closed. Stay-at-home orders have indeed a larger impact on women's mobility, especially for those aged between 25 and 44 that are more likely to have young children. Furthermore, evidence from a few regions in Northern Italy that closed schools before adopting stay-at-home orders shows that the mobility gender gap opened already at the time of school closures. In this respect, this paper contributes to the broader literature on the determinants of labor force participation. Previous studies have found that (exogenous changes in the) length of school schedules impact female labor force participation (Berthelon et al. 2015). Our study provides complementary high-frequency evidence.

These findings warn about a possible widening of gender inequality, as women may compromise their employment opportunities if they have to stay at home to care for children. These concerns are further heightened by the fact that women tend to be employed in contact-intensive sectors - such hospitality, personal care, and retail - that have been more severely impacted by the pandemic. Targeted policy intervention is required to support women during the pandemic, for example, by offering parental leave to both men and women to encourage equal burden sharing in caring for children when schools are closed.

Second, the analysis contributes to the debate about the uneven effects of the crisis across age groups. By containing the spread of the virus, lockdowns benefit 
especially people above 65 years of age because they face much greater health risks from COVID-19. The economic costs of lockdowns fall instead disproportionately on working age people. The analysis shows that lockdowns lead to a stronger reduction in the mobility of younger people, for example, by preventing them from reaching their work places and bringing children to school.

Interestingly, the mobility of younger people responds more strongly also to rising infections, for a given level of the stringency of lockdowns. Consistent with survey evidence, this could be because younger people are more concerned about the virus despite being less likely to develop severe health conditions. Or it may capture that rising infections reduce business activities in contact-intensive sectors, such as bars and restaurants, leaving many young people that work in those sectors unemployed.

The disproportionate impact of lockdowns on the mobility of the young is particularly concerning because young workers depend on labor income to sustain consumption while older people have access to larger personal saving and often receive stable retirement income. Younger workers also tend to have less stable job contracts that are more likely to be terminated during a crisis. These considerations highlight the need for a social pact across generations to at least partially compensate younger workers for the economic losses they face because of lockdowns. This is essential not only from a fairness standpoint but also to ensure enough public support to deploy lockdown measures when needed.

Third, the results on the differential effects on age groups provide insights on the possible long-term effects of the lockdown. The fact that younger generations reduced mobility more the older generations during the lockdown suggests that the scarring effects could be long lasting. This effect would be compounding the known effect that generations entering the labor force during a recession suffer a long-term scarring effect. This is a preliminary insight that should be investigated further in the future.

Fourth, the fact that different demographic groups react differently to stay-athome orders, school closures, and COVID-19 cases can provide important inputs for the formulation of targeted policies in the context of resurgent epidemics in several regions of the world. The results from this work can be useful also to inform the calibration exercises done by papers, including (Acemoglu et al. 2020; Gollier 2020), which have elaborated models with heterogeneous agents.

\section{Appendix 1: Eurostat NUTS-3 Provincial Disaggregation Illustration}

See Figs. 14 and 15. 
(a) Italy

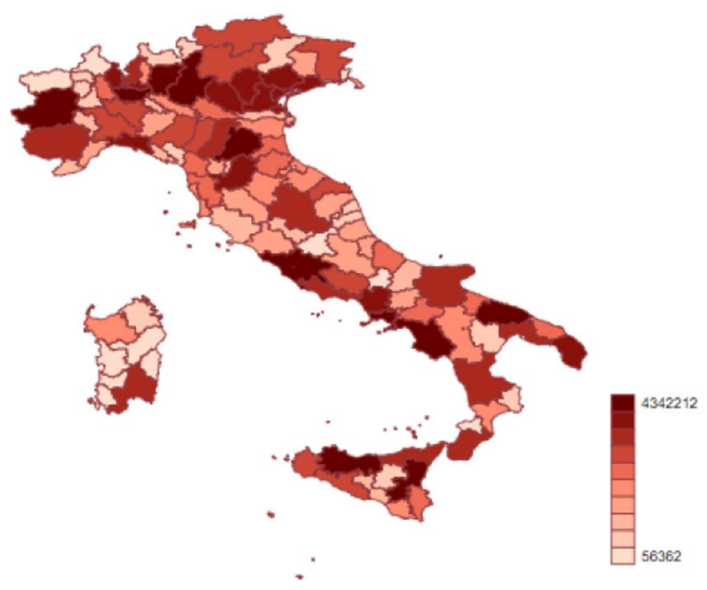

(b) Portugal

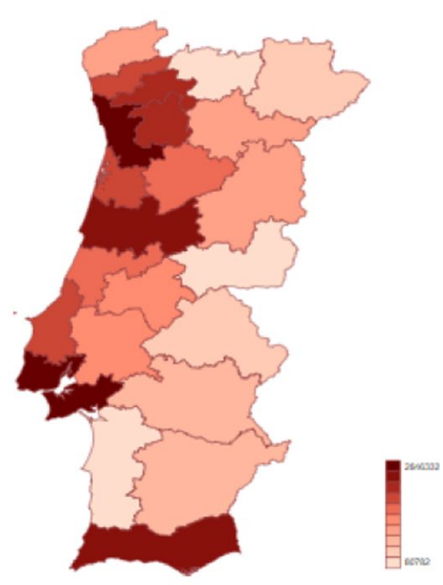

(c) Spain

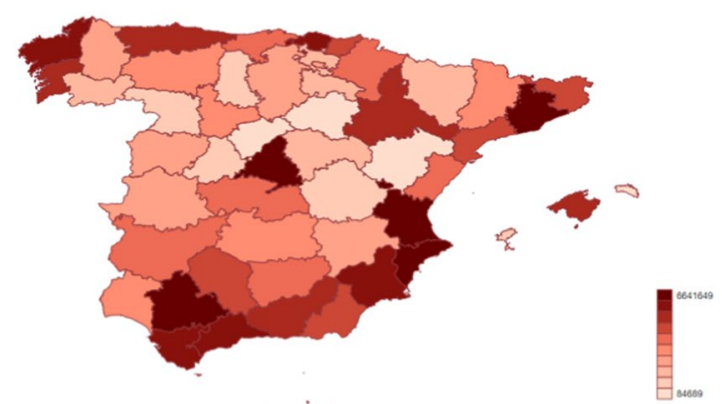

Fig. 14 Population in 2018 .Notes: the figure reports the population by NUTS-3 province across the three countries in 2018 
(a) Impact of a full lockdown on lockdown stringency

(Percentage of people moving)

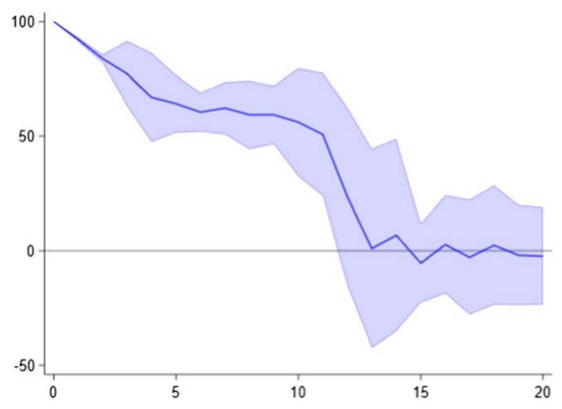

(b) Lockdown stringency (Index)

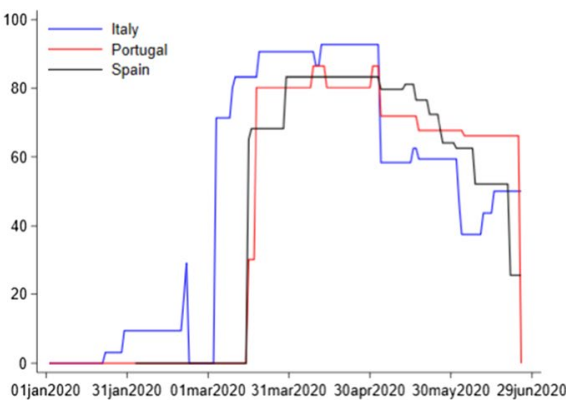

Fig. 15 Lockdown Stringency Dynamics .Notes: In panel B1a, the $x$-axis denotes the number of days, the line denotes the point estimates, and the shaded area corresponds to the 90 percent confidence interval computed with standard errors clustered at the province level

\section{Appendix 2: Lockdown Stringency Dynamics}

To better understand the dynamics uncovered by the local projections regarding how lockdowns affect mobility, it is helpful to examine how the stringency of lockdowns evolves over the local projection horizon. Panel B1a shows that a lockdown tightening tends to gradually decline and dissipate after about two weeks. These estimated dynamics reflect the way in which Italy, Portugal, and Spain have adjusted their lockdown stringency during the sample of analysis. As illustrated in panel B1b, countries have adjusted the stringency of lockdowns rather frequently.

Acknowledgements The views expressed in this working paper are those of the authors and do not necessarily representthose of the IMF, its Executive Board, or its management. Working papers describe research in progress by the authors and are published to elicit comments and to encourage debate. We thank Pedro Rente Loureco and Vodafone's Big Data and Artificial Intelligence team for constructing the mobility indicators used in this study.

\section{References}

Acemoglu, Daron, Victor Chernozhukov, Iván Werning, and Michael D. Whinston. 2020. Optimal Targeted Lockdowns in a Multi-Group SIR Model. NBER Working Paper No. 27102.

Adams, Abigail, Teodora. Boneva, Marta Golin, and Christopher Rauh. 2020. Inequality in the Impact of the Coronavirus Shock: Evidence from Real Time Surveys. CEPR Discussion Paper 14665.

Adda, Jérôme. 2016. Economic Activity and the Spread of Viral Diseases: Evidence from High Frequency Data. Quarterly Journal of Economics 131(2), 891-941.

Alon, Titan, Matthias. Doepke, Jane Olmstead-Rumsey, and Michèle Tertilt. 2020. This Time It's Different: The Role of Women's Employment in a Pandemic Recession. CEPR Discussion Paper 15149.

Anderson, Michael L. 2014. Subways, Strikes, and Slowdowns: The Impacts of Public Transit on Traffic Congestion. American Economic Review 104(9), 2763-2796. 
Aum, Sangmin, Sang. Yoon Tim Lee, and Yongseok Shin. 2020. COVID-19 Doesn't Need Lockdowns to Destroy Jobs: The Effect of Local Outbreaks in Korea. NBER Working Paper No. 27264.

Belot, Michèle, Syngjoo Choi, Egon Tripodi, Eline van den Broek-Altenburg, Julian C. Jamison, and Nicholas W. Papageorge. 2020. Unequal Consequences of COVID-19 across Age and Income: Representative Evidence from Six Countries. In Covid Economics, vol. 38.

Berthelon, Matias, Diana Kruger, and Melanie Oyarzún. 2015. The Effects of Longer School Days on Mothers' Labor Force Participation. IZA Discussion Paper No. 9212.

Bordalo, Pedro, Katherine B. Coffman, Nicola Gennaioli, and Andrei Shleifer (2020). Older People are Less Pessimistic about the Health Risks of Covid-19. NBER Working Paper No. 27494.

Chetty, Raj, John N. Friedman, Nathaniel Hendren, and Michael Stepner (2020). How Did COVID-19 and Stabilization Policies Affect Spending and Employment? A New Real-Time Economic Tracker Based on Private Sector Data. NBER Working Paper No. 27431.

Davis, Lucas W. 2008. The Effect of Driving Restrictions on Air Quality in Mexico City. Journal of Political Economy 116(1), 38-81.

Driscoll, John C and Aart C. Kraay. 1998.Consistent Covariance Matrix Estimation with Spatially Dependent Panel Data. Review of economics and statistics 80(4), 549-560.

Gertler, Mark, Huckfeldt Christopher, and Trigari Antonella. 2020. Unemployment Fluctuations, Match Quality, and the Wage Cyclicality of New Hires. The Review of Economic Studies 87(4), 1876-1914.

Glaeser, Edward L, Caitlin S Gorback, and Stephen J Redding. 2020. How Much Does COVID-19 Increase with Mobility? Evidence from New York and Four Other us Cities. NBER, Working Paper No. 27519.

Glover, Andrew, Jonathan Heathcote, Dirk Krueger, and José-Víctor Ríos-Rull. 2020. Health versus Wealth: On the Distributional Effects of Controlling a Pandemic. In Covid Economics, vol. 6, 22-64.

Gollier, Christian. 2020. Cost-Benefit Analysis of Age-Specific Deconfinement Strategies. Journal of Public Economic Theory 22(6): 1746-1771.

Goolsbee, Austan and Chad Syverson. 2020. Fear, Lockdown, and Diversion: Comparing Drivers of Pandemic Economic Decline. NBER Working Paper No. 27432.

Huckfeldt, Christopher. 2018. Understanding the Scarring Effect of Recessions. In 2018 Meeting Papers 1207, Society for Economic Dynamics.

Hupkau, Claudia and Barbara Petrangolo. 2020. Work, Care and Gender during the COVID-19 Crisis. In Covid Economics, vol. 54.

IMF. 2021. Recessions and Recoveries in Labor Markets: Patterns, Policies, and Responses to the COVID- 19 Shock. In World Economic Outlook, Chapter 3, April, International Monetary Fund.

Jordà, Òscar (2005). Estimation and Inference of Impulse Responses by Local Projections. American Economic Review 95(1): 161-182.

Lourenco, Pedro Rente, Gurjeet Kaur, Matthew Allison, and Terry Evetts. 2020. Data sharing and collaborations with Telco data during the COVID-19 pandemic: A Vodafone case study. Data \& Policy, forthcoming.

Maloney, William and Temel Taskin. 2020. Determinants of Social Distancing and Economic Activity During COVID-19: A Global View. In Covid Economics, vol. 13.

Montenovo, Laura, Xuan Jiang, Felipe Lozano Rojas, Ian M Schmutte, Kosali I Simon, Bruce A Weinberg, and Coady Wing. 2020. Determinants of Disparities in Covid-19 Job Losses. NBER Working Paper 27132.

Oreopoulos, Philip, Till Von Wachter, and Andrew Heisz. 2012. The Short-and Long-Term Career Effects of Graduating in a Recession. American Economic Journal: Applied Economics 4(1): 1-29.

Sevilla, Almudena and Sarah Smith. 2020. Baby Steps: The Gender Division of Childcare during the COVID19 Pandemic. In CEPR Covid Economics Issue vol. 23.

Publisher's Note Springer Nature remains neutral with regard to jurisdictional claims in published maps and institutional affiliations.

Francesca Caselli is an Economist at the International Monetary Fund; her email address is fcaselli@imf. org.

Francesco Grigoli is a Senior Economist at the International Monetary Fund; his email address is fgrigoli@imf.org. 
Damiano Sandri is a Deputy Division Chief at the International Monetary Fund and a research fellow at CEPR; his email address is dsandri@imf.org.

Antonio Spilimbergo is a Deputy Director at the International Monetary Fund and a research fellow at CEPR; his email address is aspilimbergo@imf.org. 